\title{
Complexity Analysis of Continuous Petri Nets`
}

\author{
Estíbaliz Fraca $^{1}$, Serge Haddad ${ }^{2}$ \\ 1 Instituto de Investigación en Ingeniería de Aragón (I3A) \\ Universidad de Zaragoza, Zaragoza, Spain \\ E-mail: efraca@unizar.es \\ 2 Ecole Normale Supérieure de Cachan, \\ LSV, CNRS UMR 8643, INRIA, Cachan, France \\ E-mail: haddad@lsv.ens-cachan.fr
}

\begin{abstract}
At the end of the eighties, continuous Petri nets were introduced for: (1) alleviating the combinatory explosion triggered by discrete Petri nets and, (2) modelling the behaviour of physical systems whose state is composed of continuous variables. Since then several works have established that the computational complexity of deciding some standard behavioural properties of Petri nets is reduced in this framework. Here we first establish the decidability of additional properties like boundedness and reachability set inclusion. We also design new decision procedures for the reachability and lim-reachability problems with a better computational complexity. Finally we provide lower bounds characterising the exact complexity class of the boundedness, the reachability, the deadlock freeness and the liveness problems.
\end{abstract}

\section{Introduction}

From Petri nets to continuous Petri nets. Continuous Petri nets (CPN) were introduced in [5] by considering continuous states (specified by a non negative real number of tokens in places) where the dynamics of the system is triggered either by discrete events or by a continuous evolution ruled by speed of firings. In the former case such nets are called autonomous CPNs while in the latter they are called timed CPNs. In both cases, the evolution is due to a fractional transition firing (infinitesimal and simultaneous in the case of timed CPNs).

Modelling with CPNs. CPNs have been used in several significant application fields. In [3], a method based on CPNs is proposed for the fault diagnosis of manufacturing systems that manage systems intractable with discrete Petri nets (for modelling of manufacturing systems see also [16]). In [14], the authors introduce a bottom-up modelling methodology based on CPNs to represent cell metabolism and solve in this framework the regulation control problem. Combining discrete and continuous Petri nets yields hybrid Petri nets with applications

\footnotetext{
* This work has been partially supported by CICYT - FEDER project DPI2010-20413, by a pre-doctoral grant of the Gobierno de Aragón and by Fundación Aragón I+D and by ImpRo (ANR-2010-BLAN-0317).
} 
to modelling and simulation of water distribution systems [8] and to the analysis of traffic in urban networks [15].

Analysis of CPNs. While several analysis methods have been developed for timed CPNs there is no hope for fully automatic techniques in the general case since standard problems of dynamic systems are known to be undecidable even for bounded nets [12].

Due to the semantics of autonomous CPNs, a marking can be the limit of the markings visited along an infinite firing sequence. Thus most of the usual properties are duplicated depending on whether these markings are considered or not. When considering these markings, reachability (resp. liveness, deadlockfreeness) becomes lim-reachability (resp. lim-liveness, lim-deadlock-freeness).

Contrary to the timed case, the analysis of autonomous CPNs (that we simply call CPNs in the sequel) appears to be less complex than the one of discrete Petri nets. In [9], exponential time decision procedures are proposed for the reachability and lim-reachability problems for general CPNs. In [13] assuming additional hypotheses on the net, the authors design polynomial time decision procedures for (lim-)reachability and boundedness. In [12], (lim-)deadlock-freeness and (lim-)liveness are shown to belong in coNP. These procedures are based on "simple" characterisations of the properties.

Our contributions. First we revisit characterisations of properties in CPN establishing an alternative characterisation for reachability and the first characterisation for boundedness. Then based on these characterisations, we show that (lim-)reachability and boundedness are decidable in polynomial time. We also establish that the (lim-)reachability set inclusion problem is decidable in exponential time. Finally we prove that (lim-)reachability and boundedness are PTIMEhard and that (lim-)deadlock-freeness, (lim-)liveness and (lim-)reachability set inclusion problems are coNP-hard. We establish these lower bounds even when considering restricted cases of these problems.

Organisation. In Section 2, we introduce CPNs and the properties that we are analysing. In Section 3, we develop the characterisations of reachability and boundedness. Afterwards in Section 4, we design the decision procedures. Then, we provide complexity lower bounds in Section 5. Finally in Section 6, we summarise our results and give perspectives to this work. Two hardness proofs are given in appendix and will be omitted in the final version.

\section{Continuous Petri nets: definitions and properties}

\subsection{Continuous Petri nets}

Notations. $\mathbb{N}($ resp. $\mathbb{Q}, \mathbb{R}$ ) is the set of non negative integers (resp. rational, real numbers). Given a set of numbers $E, E_{\geq 0}$ (resp. $E_{>0}$ ) denotes the subset of non negative (resp. positive) numbers of $E$. Given an $E \times F$ matrix $\mathbf{M}$ with $E$ and $F$ sets of indices, $E^{\prime} \subseteq E$ and $F^{\prime} \subseteq F$, the $E^{\prime} \times F^{\prime}$ submatrix $\mathbf{M}_{E^{\prime} \times F^{\prime}}$ denotes the restriction of $\mathbf{M}$ to rows indexed by $E^{\prime}$ and columns indexed by $F^{\prime}$. The support 
of a vector $\mathbf{v} \in \mathbb{R}^{E}$, denoted $\llbracket \mathbf{v} \rrbracket$, is defined by $\llbracket \mathbf{v} \rrbracket \stackrel{\text { def }}{=}\{e \in E \mid \mathbf{v}[e] \neq 0\}$. $\mathbf{0}$ denotes the null vector. One writes $\mathbf{v} \geq \mathbf{w}$ when $\mathbf{v}$ is componentwise greater or equal than $\mathbf{w}$ and $\mathbf{v} \geq \mathbf{w}$ when $\mathbf{v} \geq \mathbf{w}$ and $\mathbf{v} \neq \mathbf{w}$. One writes $\mathbf{v}>\mathbf{w}$ when $\mathbf{v}$ is componentwise strictly greater than $\mathbf{w}$. $\|\mathbf{v}\|_{1}$ is the 1-norm of $\mathbf{v}$ defined by $\|\mathbf{v}\|_{1} \stackrel{\text { def }}{=} \sum_{e \in E}|\mathbf{v}[e]|$. Let $E^{\prime} \subseteq E$, then $\mathbf{v}\left[E^{\prime}\right]$ denotes the restriction of $\mathbf{v}$ to components of $E^{\prime}$.

Here, we adopt the following terminology: a net denotes the structure without initial marking while a net system denotes a net with an initial marking. The structure of CPNs and discrete nets are identical.

Definition 1 A Petri net $(P N)$ is a tuple $\mathcal{N}=\langle P, T$, Pre, Post $\rangle$ where:

- $P$ is a finite set of places;

- $T$ is a finite set of transitions, with $P \cap T=\emptyset$;

- Pre (resp. Post), is the backward (resp. forward) $P \times T$ incidence matrix, whose items belong to $\mathbb{N}$.

The incidence matrix $\boldsymbol{C}$ is defined by $\boldsymbol{C} \stackrel{\text { def }}{=} \boldsymbol{P o s t}-\boldsymbol{P r} \boldsymbol{e}$.

Given a place (resp. transition) $v$ in $P$ (resp. in $T$ ), its preset, $\bullet v$, is defined as the set of its input transitions (resp. places): $\bullet \stackrel{\text { def }}{=}\{t \in T \mid \boldsymbol{P o s t}[v, t]>0\}$ (resp. $\left.{ }^{\bullet} v \stackrel{\text { def }}{=}\{p \in P \mid \boldsymbol{P r} \boldsymbol{e}[p, v]>0\}\right)$. Its postset $v^{\bullet}$ is defined as the set of its output transitions (resp. places): $v^{\bullet} \stackrel{\text { def }}{=}\{t \in T \mid \operatorname{Pr} \boldsymbol{e}[v, t]>0\}$ (resp. $\left.v^{\bullet} \stackrel{\text { def }}{=}\{p \in P \mid \boldsymbol{P o s t}[p, v]>0\}\right)$. This notion generalizes to a subset $V$ of places (resp. transitions) by: ${ }^{\bullet} V \stackrel{\text { def }}{=} \bigcup_{v \in V} \bullet$ and $V^{\bullet} \stackrel{\text { def }}{=} \bigcup_{v \in V} v^{\bullet}$. In addition, $\bullet V^{\bullet} \stackrel{\text { def }}{=} \bullet V \cup V^{\bullet}$.

Given $T^{\prime} \subseteq T, \mathcal{N}_{T^{\prime}}$ is the subnet of $\mathcal{N}$ such that its set of transitions is $T^{\prime}$ and its set of places is $\boldsymbol{\bullet}^{\mathbf{\bullet}}$, and its backward and forward incidence matrices are respectively $\boldsymbol{P r} \boldsymbol{e} \cdot T^{\prime} \bullet \times T^{\prime}$ and $\boldsymbol{P o s t}_{T^{\prime}} \bullet \times T^{\prime}$.

We define $\mathcal{N}^{-1}$ as the "reverse" net of $\mathcal{N}$, in which the places and transitions coincide, and its arcs are inverted.

Definition 2 Given a $P N \mathcal{N}=\langle P, T$, Pre, Post $\rangle$, its reverse net $\mathcal{N}^{-1}$ is defined by $\mathcal{N}^{-1} \stackrel{\text { def }}{=}\langle P, T$, Post, Pre $\rangle$.

A continuous PN system consists of a net and a non negative real marking.

Definition 3 A CPN system is a tuple $\left\langle\mathcal{N}, \boldsymbol{m}_{0}\right\rangle$ where $\mathcal{N}$ is a $P N$ and $\boldsymbol{m}_{0} \in$ $\mathbb{R}_{\geq 0}^{P}$ is the initial marking.

When a CPN system is an input of a decision problem, the items of $\boldsymbol{m}_{0}$ are rational numbers in order to characterise the complexity of the problem.

In discrete PNs the firing rule of a transition requires tokens specified by $\boldsymbol{P r}$ e to be present in the corresponding places. In continuous PNs a non negative real amount of transition firing is allowed and this amount scales the requirement expressed by Pre and Post. 
Definition 4 Let $\mathcal{N}$ be a CPN, $t$ be a transition and $\boldsymbol{m} \in \mathbb{R}_{\geq 0}^{P}$ be a marking.

- The enabling degree of $t$ w.r.t. $\boldsymbol{m}$, enab $(t, \boldsymbol{m}) \in \mathbb{R}_{\geq 0} \cup \infty$, is defined by: $\operatorname{enab}(t, \boldsymbol{m}) \stackrel{\text { def }}{=} \min \left\{\frac{\boldsymbol{m}[p]}{\boldsymbol{P r} \boldsymbol{e}[p, t]} \mid p \in \bullet t\right\} \quad(\operatorname{enab}(t, \boldsymbol{m})=\infty$ iff $\bullet t=\emptyset)$.

$-t$ is enabled in $\boldsymbol{m}$ if enab $(t, \boldsymbol{m})>0$.

- $t$ can be fired by any amount $\alpha \in \mathbb{R}$ such that $t^{3} 0 \leq \alpha \leq$ enab $(t, \boldsymbol{m})$, and its firing leads to marking $\boldsymbol{m}^{\prime}$ defined by: for all $p \in P, \boldsymbol{m}^{\prime}[p]=\boldsymbol{m}[p]+\alpha \boldsymbol{C}[p, t]$.

The firing of $t$ from $\boldsymbol{m}$ by an amount $\alpha$ leading to $\boldsymbol{m}^{\prime}$ is denoted as $\boldsymbol{m} \stackrel{\alpha t}{\longrightarrow} \boldsymbol{m}^{\prime}$. We illustrate the firing rule of a CPN with the system in Fig. 1(a) (example taken from [9]). In the initial marking $\boldsymbol{m}_{0}=(1,0,1,0)$, only transition $t_{1}$ is enabled and its enabling degree is 1 . Hence, it can be fired by any real amount $\alpha$ s.t. $0 \leq \alpha \leq 1$. If $t_{1}$ is fired by an amount of 0.5 , marking $\boldsymbol{m}_{1}=(0.5,0.5,1,0)$ is reached. In $\boldsymbol{m}_{1}$, transitions $t_{1}$ and $t_{2}$ are enabled, with enabling degree both equal to 0.5 .

Let $\sigma=\alpha_{1} t_{1} \ldots \alpha_{n} t_{n}$ be a finite sequence with for all $i, t_{i} \in T$ and $\alpha_{i} \in \mathbb{R}_{\geq 0}$. $\sigma$ is firable from $\boldsymbol{m}_{0}$ if for all $1 \leq i \leq n$ there exist $\boldsymbol{m}_{i}$ such that $\boldsymbol{m}_{i-1} \stackrel{\alpha_{i} t_{i}}{\longrightarrow} \boldsymbol{m}_{i}$. This firing is denoted by $\boldsymbol{m}_{0} \stackrel{\sigma}{\longrightarrow} \boldsymbol{m}_{n}$. When the destination marking is irrelevant we omit it and simply write $\boldsymbol{m}_{0} \stackrel{\sigma}{\longrightarrow}$. Let $\sigma=\alpha_{1} t_{1} \ldots \alpha_{n} t_{n} \ldots$ be an infinite sequence then $\sigma$ is firable from $\boldsymbol{m}_{0}$ if for all $n, \alpha_{1} t_{1} \ldots \alpha_{n} t_{n}$ is firable from $\boldsymbol{m}_{0}$. This firing is denoted as $\boldsymbol{m}_{0} \stackrel{\sigma}{\longrightarrow}$.

Given a finite or infinite sequence $\sigma=\alpha_{1} t_{1} \ldots \alpha_{i} t_{i} \ldots$ and $\alpha \in \mathbb{R}_{\geq 0}$, the sequence $\alpha \sigma$ is defined by $\sigma \stackrel{\text { def }}{=} \alpha \alpha_{1} t_{1} \ldots \alpha \alpha_{i} t_{i} \ldots$ Given two infinite sequences $\sigma=\alpha_{1} t_{1} \ldots \alpha_{i} t_{i} \ldots$ and $\sigma^{\prime}=\alpha_{1}^{\prime} t_{1}^{\prime} \ldots \alpha_{i}^{\prime} t_{i}^{\prime} \ldots$, the (non commutative) sum $\sigma+$ $\sigma^{\prime}$ is defined by: $\sigma+\sigma^{\prime} \stackrel{\text { def }}{=} \alpha_{1} t_{1} \alpha_{1}^{\prime} t_{1}^{\prime} \ldots \alpha_{i} t_{i} \alpha_{i}^{\prime} t_{i}^{\prime} \ldots$ This notion generalises to arbitrary sequences by extending them to infinite sequences with null amounts of firings (the selected transitions are irrelevant).

Let $\sigma=\alpha_{1} t_{1} \ldots \alpha_{n} t_{n}$ be a finite sequence and denote $\sigma^{-1}=\alpha_{n} t_{n} \ldots \alpha_{1} t_{1}$. By definition of the reverse net, $\boldsymbol{m} \stackrel{\sigma}{\longrightarrow} \boldsymbol{m}^{\prime}$ in $\mathcal{N}$ iff $\boldsymbol{m}^{\prime} \stackrel{\sigma^{-1}}{\longrightarrow} \boldsymbol{m}$ in $\mathcal{N}^{-1}$.

The Parikh image (also called firing count vector) of a (finite or infinite) firing sequence $\sigma=\alpha_{1} t_{1} \ldots \alpha_{n} t_{n} \ldots$ denoted $\vec{\sigma} \in\left(\mathbb{R}_{\geq 0} \cup\{\infty\}\right)^{T}$ is defined by: $\vec{\sigma}[t] \stackrel{\text { def }}{=} \sum_{i \mid t_{i}=t} \alpha_{i}$. As in discrete PNs, when $\boldsymbol{m} \stackrel{\sigma}{\longrightarrow} \boldsymbol{m}^{\prime}, \boldsymbol{m}^{\prime}=\boldsymbol{m}+\boldsymbol{C} \vec{\sigma}$ and this equation is called the state equation.

A set of places $P^{\prime}$ is a siphon if ${ }^{\bullet} P^{\prime} \subseteq P^{\prime} \bullet$. When a siphon does not contain tokens in some marking, it will never contain tokens after any firing sequence starting from this marking. One call it an empty siphon.

An interesting difference between discrete and continuous PN systems is that the sequence of markings visited by an infinite firing sequence may converge to a given marking. For example, let us consider again the CPN of Fig. 1(a), and the marking $\boldsymbol{m}_{1}=(0.5,0.5,1,0)$. From $\boldsymbol{m}_{1}, 0.5 t_{2}$ can be fired, reaching $\boldsymbol{m}_{2}=(0.5,0.5,0,0.5)$. From $\boldsymbol{m}_{2}$ transition $t_{3}$ can be fired by an amount of 0.5 ,

\footnotetext{
${ }^{3}$ So from every marking, any (even disabled) transition can fire by a null amount without modifying the marking.
} 


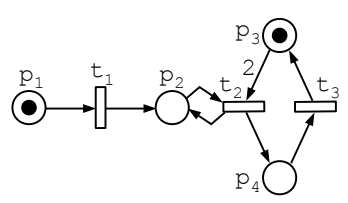

(a)

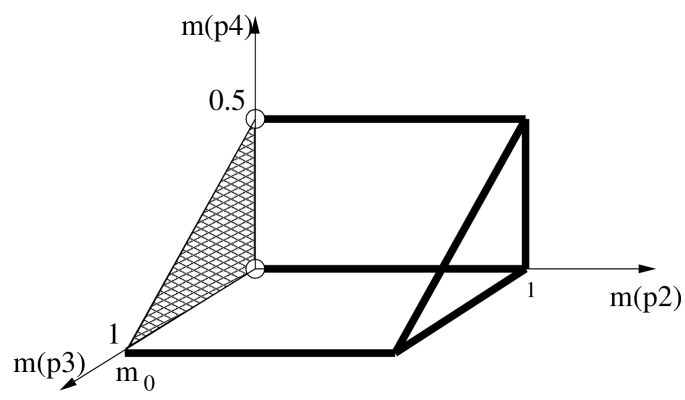

(b)

Fig. 1. (a) A CPN system (b) its lim-reachability set [9]

leading to $\boldsymbol{m}_{3}=(0.5,0.5,0.25,0)$. Iterating this process leads to the infinite firing sequence $\sigma=2^{-1} t_{2} 2^{-1} t_{3} \ldots 2^{-n} t_{2} 2^{-n} t_{3} \ldots$ whose visited markings converge toward $(0.5,0.5,0,0)$. Observe that the Parikh image $\vec{\sigma}=\overrightarrow{t_{2}}+\overrightarrow{t_{3}}$ does not correspond to any finite firing sequence starting from $\boldsymbol{m}_{1}$.

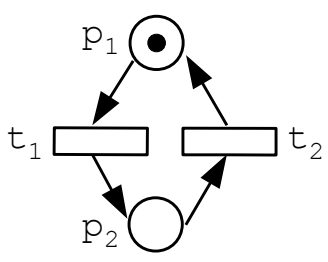

Fig. 2. A simple CPN system.

Consider now the PN in Fig. 2 with initial marking $\boldsymbol{m}_{0}=(1,0)$. Let $\sigma=$ $1 t_{1} \frac{1}{2} t_{2} \frac{1}{3} t_{1} \frac{1}{4} t_{2} \ldots \frac{1}{2 i-1} t_{1} \frac{1}{2 i} t_{2} \ldots$ The sequence $\sigma$ is infinite and its sequence of visited markings converges toward marking $\boldsymbol{m}$ defined by: $\boldsymbol{m} \stackrel{\text { def }}{=}(1-\log (2), \log (2))$. Here $\vec{\sigma}=\infty \overrightarrow{t_{1}}+\infty \overrightarrow{t_{2}}$.

Let $\sigma$ be an infinite firing sequence starting from $\boldsymbol{m}$ whose sequence of visited markings converges toward $\boldsymbol{m}^{\prime}$, one says that $\boldsymbol{m}^{\prime}$ is limit reachable from $\boldsymbol{m}$ which 
is denoted by: $\boldsymbol{m} \stackrel{\sigma}{\longrightarrow} \boldsymbol{m}^{\prime}$. Thus in CPNs, two sets of reachable markings are defined.

Definition 5 Given a $C P N$ system $\left\langle\mathcal{N}, \boldsymbol{m}_{0}\right\rangle$,

- Its reachability set $\operatorname{RS}\left(\mathcal{N}, \boldsymbol{m}_{0}\right)$ is defined by: $\operatorname{RS}\left(\mathcal{N}, \boldsymbol{m}_{0}\right) \stackrel{\text { def }}{=}\left\{\boldsymbol{m} \mid\right.$ there exists a finite sequence $\left.\boldsymbol{m}_{0} \stackrel{\sigma}{\longrightarrow} \boldsymbol{m}\right\}$.

- Its lim-reachability set, $\lim -\operatorname{RS}\left(\mathcal{N}, \boldsymbol{m}_{0}\right)$, is defined by: $\lim -\operatorname{RS}\left(\mathcal{N}, \boldsymbol{m}_{0}\right) \stackrel{\text { def }}{=}\left\{\boldsymbol{m} \mid\right.$ there exists an infinite sequence $\left.\boldsymbol{m}_{0} \stackrel{\sigma}{\longrightarrow} \boldsymbol{m}\right\}$.

RS or lim-RS are convex sets (see Section 3) but not necessarily topologically closed. In Fig. 1, marking $\boldsymbol{m}=(1,0,0,0)$ belongs to the closure of RS or lim-RS, but it does not belong to these sets. Since an infinite sequence can include null amounts of firings, $\operatorname{RS}\left(\mathcal{N}, \boldsymbol{m}_{0}\right) \subseteq \lim -\operatorname{RS}\left(\mathcal{N}, \boldsymbol{m}_{0}\right)$. More interestingly, for all $\boldsymbol{m} \in \lim -\operatorname{RS}\left(\mathcal{N}, \boldsymbol{m}_{0}\right), \lim -\operatorname{RS}(\mathcal{N}, \boldsymbol{m}) \subseteq \lim -\operatorname{RS}\left(\mathcal{N}, \boldsymbol{m}_{0}\right)$ (see the proof in appendix). So there is no need to consider iterations of lim-reachability.

\section{$2.2 \quad$ CPN properties}

Here we introduce the standard properties that a modeller wants to check on a net. In the framework of CPNs, every property its defined either w.r.t. to the reachability set or w.r.t. to the lim-reachability set.

Reachability is the main property as it is the core of safeness properties.

Definition 6 (reachability) Given a system $\left\langle\mathcal{N}, \boldsymbol{m}_{0}\right\rangle$ and a marking $\boldsymbol{m}, \boldsymbol{m}$ is (lim-)reachable in $\left\langle\mathcal{N}, \boldsymbol{m}_{0}\right\rangle$ if $\boldsymbol{m} \in(\lim -) \operatorname{RS}\left(\mathcal{N}, \boldsymbol{m}_{0}\right)$.

Boundedness is often related to the resources needed by the system. For CPN, boundedness and lim-boundedness coincide [13].

Definition 7 (boundedness) A system $\left\langle\mathcal{N}, \boldsymbol{m}_{0}\right\rangle$ is (lim-)bounded if there exists $b \in \mathbb{R}_{\geq 0}$ such that for all $\boldsymbol{m} \in(\lim -) \operatorname{RS}\left(\mathcal{N}, \boldsymbol{m}_{0}\right)$ and all $p \in P, \boldsymbol{m}[p] \leq b$.

Deadlock-freeness ensures that a system will never reach a marking where no transition is enabled, i.e a dead marking.

Definition 8 (deadlock-freeness) A system $\left\langle\mathcal{N}, \boldsymbol{m}_{0}\right\rangle$ is (lim-)deadlock-free if for all $\boldsymbol{m} \in(\lim -) \operatorname{RS}\left(\mathcal{N}, \boldsymbol{m}_{0}\right)$, there exists $t \in T$ such that $t$ is enabled at $\boldsymbol{m}$.

The net of Fig. 1 is deadlock-free but not lim-deadlock-free: $\boldsymbol{m} \stackrel{\text { def }}{=}(0,1,0,0)$ is a dead marking which is limit-reachable but not reachable and no reachable marking is dead.

Liveness ensures that whatever the reachable state, any transition will be fireable in some future. So the system never "looses its capacities".

Definition 9 (liveness) A system $\left\langle\mathcal{N}, \boldsymbol{m}_{0}\right\rangle$ is (lim-)live if for all transition $t$ and for all marking $\boldsymbol{m} \in(\lim -) \operatorname{RS}\left(\mathcal{N}, \boldsymbol{m}_{0}\right)$ there exists $\boldsymbol{m}^{\prime} \in(\lim -) \operatorname{RS}(\mathcal{N}, \boldsymbol{m})$ such that $t$ is enabled at $\boldsymbol{m}^{\prime}$. 
The net of Fig. 1 is neither live nor lim-live: once $t_{1}$ becomes disabled, it will remain so whatever the finite or infinite firing sequence considered.

A home state is a marking that can be reached whatever the current state. This property can express for instance that recovering from faults is always possible. A net is reversible if its initial marking is an home state. Both properties are particular cases of the reachability set inclusion problem.

\section{Definition 10 (reachability set inclusion)}

Given systems $\left\langle\mathcal{N}, \boldsymbol{m}_{0}\right\rangle$ and $\left\langle\mathcal{N}^{\prime}, \boldsymbol{m}_{0}^{\prime}\right\rangle$ with $P=P^{\prime},\left\langle\mathcal{N}, \boldsymbol{m}_{0}\right\rangle$ is (lim-)reachable included in $\left\langle\mathcal{N}^{\prime}, \boldsymbol{m}_{0}^{\prime}\right\rangle$ if $(\mathrm{lim}-) \operatorname{RS}\left(\mathcal{N}, \boldsymbol{m}_{0}\right) \subseteq(\lim -) \operatorname{RS}\left(\mathcal{N}^{\prime}, \boldsymbol{m}_{0}^{\prime}\right)$.

A marking $\boldsymbol{m}$ is a home state if $\operatorname{RS}\left(\mathcal{N}, \boldsymbol{m}_{0}\right) \subseteq \operatorname{RS}\left(\mathcal{N}^{-1}, \boldsymbol{m}\right)$.

When $\boldsymbol{m}=\boldsymbol{m}_{0}$, one says that $\left\langle\mathcal{N}, \boldsymbol{m}_{0}\right\rangle$ is reversible.

The following table summarises the results already known about the complexity of the associated decision problems. A net is consistent if there exists a vector $\mathbf{v} \in \mathbb{R}_{\geq 0}$ with $\llbracket v \rrbracket=T$ and $\boldsymbol{C} \mathbf{v}=0$. No lower bounds have been established.

Table 1. Complexity bounds: previous results

\begin{tabular}{|l|l|}
\hline \multicolumn{1}{|c|}{ Problems } & \multicolumn{1}{c|}{ Upper bounds } \\
\hline (lim-)reachability & $\begin{array}{l}\text { in EXPTIME [9] } \\
\text { in PTIME for lim-reachability } \\
\text { when all transitions are fireable at least once } \\
\text { and the net is consistent [13] }\end{array}$ \\
\hline (lim-)boundedness & $\begin{array}{l}\text { in PTIME } \\
\text { when all transitions are fireable at least once [13] }\end{array}$ \\
\hline (lim-)deadlock-freeness & in coNP [12] \\
\hline (lim-)liveness & in coNP [12] \\
\hline $\begin{array}{l}\text { (lim-)reachability } \\
\text { set inclusion }\end{array}$ & no result \\
\hline
\end{tabular}

\section{Properties characterisations}

\subsection{Preliminary results about reachability and firing sequences}

Most of the results of this subsection are generalisations of results given in [13, 9].

The following lemma is an almost immediate consequence of firing definition and has for corollary the convexity of the (lim-)reachability set. In this lemma depending on the sequences $\longrightarrow(\infty)$ denotes either $\longrightarrow$ or $\longrightarrow_{\infty}$. 
Lemma 11 Given a CPN system $\left\langle\mathcal{N}, \boldsymbol{m}_{0}\right\rangle$, (finite or infinite) sequences $\sigma, \sigma_{1}, \sigma_{2}$ markings $\boldsymbol{m}, \boldsymbol{m}^{\prime}, \boldsymbol{m}_{1}, \boldsymbol{m}_{2}, \boldsymbol{m}_{1}^{\prime}, \boldsymbol{m}_{2}^{\prime}$ and $\alpha, \alpha_{1}, \alpha_{2} \in \mathbb{R}_{>0}$ :

(0) $\boldsymbol{m}_{1} \stackrel{\sigma}{\longrightarrow} \boldsymbol{m}_{1}^{\prime}$ and $\boldsymbol{m}_{1} \leq \boldsymbol{m}_{2}$ implies $\boldsymbol{m}_{2} \stackrel{\sigma}{\longrightarrow} \boldsymbol{m}_{2}^{\prime}$ with $\boldsymbol{m}_{1}^{\prime} \leq \boldsymbol{m}_{2}^{\prime}$

(1) $\boldsymbol{m} \stackrel{\sigma}{\longrightarrow}(\infty) \boldsymbol{m}$ iff $\alpha \boldsymbol{m} \stackrel{\alpha \sigma}{\longrightarrow}(\infty) \alpha \boldsymbol{m}^{\prime}$

(2) $\boldsymbol{m} \stackrel{\sigma}{\longrightarrow}$ iff $\alpha \boldsymbol{m} \stackrel{\alpha \sigma}{\longrightarrow} \infty$

(3) $\boldsymbol{m}_{1} \stackrel{\sigma_{1}}{\longrightarrow}(\infty) \boldsymbol{m}_{1}^{\prime}$ and $\boldsymbol{m}_{2} \stackrel{\sigma_{2}}{\longrightarrow}(\infty) \boldsymbol{m}_{2}^{\prime}$ implies $\boldsymbol{m}_{1}+\boldsymbol{m}_{2} \stackrel{\sigma_{1}+\sigma_{2}}{\longrightarrow}(\infty) \boldsymbol{m}_{1}^{\prime}+\boldsymbol{m}_{2}^{\prime}$

(4) $\boldsymbol{m}_{1} \stackrel{\sigma_{1}}{\longrightarrow} \infty$ and $\boldsymbol{m}_{2} \stackrel{\sigma_{2}}{\longrightarrow} \infty$ implies $\boldsymbol{m}_{1}+\boldsymbol{m}_{2} \stackrel{\sigma_{1}+\sigma_{2}}{\longrightarrow} \infty$

(5) $\boldsymbol{m}_{1} \stackrel{\alpha_{1} \sigma}{\longrightarrow}(\infty) \boldsymbol{m}_{1}^{\prime}$ and $\boldsymbol{m}_{2} \stackrel{\alpha_{2} \sigma}{\longrightarrow}(\infty) \boldsymbol{m}_{2}^{\prime}$ implies $\boldsymbol{m}_{1}+\boldsymbol{m}_{2} \stackrel{\left(\alpha_{1}+\alpha_{2}\right) \sigma}{\longrightarrow}{ }_{(\infty)} \boldsymbol{m}_{1}^{\prime}+\boldsymbol{m}_{2}^{\prime}$

(6) $\boldsymbol{m}_{1} \stackrel{\alpha_{1} \sigma}{\longrightarrow}$ and $\boldsymbol{m}_{2} \stackrel{\alpha_{2} \sigma}{\longrightarrow}$ implies $\boldsymbol{m}_{1}+\boldsymbol{m}_{2} \stackrel{\left(\alpha_{1}+\alpha_{2}\right) \sigma}{\longrightarrow} \infty$

The two next lemmas constitute a first step for the characterisation of reachability since they provide sufficient conditions for reachability and lim-reachability in particular cases.

Lemma 12 Let $\left\langle\mathcal{N}, \boldsymbol{m}_{0}\right\rangle$ be a continuous system, $\boldsymbol{m}$ be a marking and $\mathbf{v} \in \mathbb{R}_{\geq 0}^{T}$ that fulfill:

$-\boldsymbol{m}=\boldsymbol{m}_{0}+\boldsymbol{C} \mathbf{v}$

$-\forall p \in \bullet \llbracket \mathbf{v} \rrbracket \boldsymbol{m}_{0}[p]>0$

$-\forall p \in \llbracket \mathbf{v} \rrbracket^{\bullet} \boldsymbol{m}[p]>0$.

Then there exists a finite sequence $\sigma$ such that $\boldsymbol{m}_{0} \stackrel{\sigma}{\longrightarrow} \boldsymbol{m}$ and $\vec{\sigma}=\mathbf{v}$.

Proof. Define $\alpha_{1} \stackrel{\text { def }}{=} \min \left(\frac{\boldsymbol{m}_{0}[p]}{\sum_{t \in \llbracket v \rrbracket} \boldsymbol{P r e}[p, t] \mathbf{v}[t]} \mid p \in \bullet \llbracket \mathbf{v} \rrbracket\right)$

and $\alpha_{2} \stackrel{\text { def }}{=} \min \left(\frac{\boldsymbol{m}[p]}{\sum_{t \in \llbracket v \rrbracket} \boldsymbol{P o s t}[p, t] \mathbf{v}[t]} \mid p \in \llbracket \mathbf{v} \rrbracket^{\bullet}\right)$ with the convention that $\alpha_{1} \stackrel{\text { def }}{=} 1$ (resp. $\alpha_{2} \stackrel{\text { def }}{=} 1$ ) if $\bullet \llbracket \mathbf{v} \rrbracket$ (resp. $\llbracket \mathbf{v} \rrbracket^{\bullet}$ ) is empty.

Due to the second and the third hypotheses $\alpha_{1}$ and $\alpha_{2}$ are positive.

Let $n \stackrel{\text { def }}{=} \max \left(\left\lceil\frac{1}{\min \left(\alpha_{1}, \alpha_{2}\right)}\right\rceil, 2\right)$.

Denote $\llbracket v \rrbracket \stackrel{\text { def }}{=}\left\{t_{1}, \ldots, t_{k}\right\}$ and define $\sigma^{\prime} \stackrel{\text { def }}{=} \frac{\mathbf{v}\left[t_{1}\right]}{n} t_{1} \ldots \frac{\mathbf{v}\left[t_{k}\right]}{n} t_{k}$ and $\sigma \stackrel{\text { def }}{=} \sigma^{\prime n}$.

We claim that $\sigma$ is the required firing sequence.

Let us denote $\boldsymbol{m}_{i} \stackrel{\text { def }}{=} \boldsymbol{m}_{0}+\frac{i}{n} \boldsymbol{C} \mathbf{v}$. Thus $\boldsymbol{m}=\boldsymbol{m}_{n}$.

By definition of $\alpha_{1}$ and $n$, in $\mathcal{N} \boldsymbol{m}_{0} \stackrel{\sigma^{\prime}}{\longrightarrow} \boldsymbol{m}_{1}$ and by definition of $\alpha_{2}, \boldsymbol{m}_{n} \stackrel{\sigma^{\prime-1}}{\longrightarrow} \boldsymbol{m}_{n-1}$ in $\mathcal{N}^{-1}$. So in $\mathcal{N} \boldsymbol{m}_{n-1} \stackrel{\sigma^{\prime}}{\longrightarrow} \boldsymbol{m}_{n}$.

Let $1<i<n-1$.

Using lemma 11, $\frac{n-1-i}{n-1} \boldsymbol{m}_{0} \stackrel{\frac{n-1-i}{n-1} \sigma^{\prime}}{\longrightarrow} \frac{n-1-i}{n-1} \boldsymbol{m}_{1}$ and $\frac{i}{n-1} \boldsymbol{m}_{n-1} \stackrel{\frac{i}{n-1} \sigma^{\prime}}{\longrightarrow} \frac{i}{n-1} \boldsymbol{m}_{n}$.

Using lemma 11 again and summing, one gets: $\boldsymbol{m}=\boldsymbol{m}_{i} \stackrel{\sigma^{\prime}}{\longrightarrow} \boldsymbol{m}_{i+1}$.

Lemma 13 Let $\left\langle\mathcal{N}, \boldsymbol{m}_{0}\right\rangle$ be a continuous system, $\boldsymbol{m}$ be a marking and $\mathbf{v} \in \mathbb{R}_{\geq 0}^{T}$ that fulfill:

$-\boldsymbol{m}=\boldsymbol{m}_{0}+\boldsymbol{C} \mathbf{v}$

$-\forall p \in \bullet \llbracket \mathbf{v} \rrbracket^{\bullet} \boldsymbol{m}_{0}[p]>0$. 
Then there exists an infinite sequence $\sigma$ such that $\boldsymbol{m}_{0} \stackrel{\sigma}{\longrightarrow} \boldsymbol{m}$ and $\vec{\sigma}=\mathbf{v}$.

Proof. Let $\boldsymbol{m}_{i}$ be inductively defined by $\boldsymbol{m}_{i+1}=\frac{1}{2} \boldsymbol{m}_{i}+\frac{1}{2} \boldsymbol{m}$. and for $i \geq 1$, let $\mathbf{v}_{i}=\frac{1}{2^{i}} \mathbf{v}$ (thus $\llbracket \mathbf{v}_{i} \rrbracket=\llbracket \mathbf{v} \rrbracket$ ). Observe that $\boldsymbol{m}_{i}=\frac{1}{2^{i}} \boldsymbol{m}_{0}+\left(1-\frac{1}{2^{i}}\right) \boldsymbol{m}$. So:

$-\boldsymbol{m}_{i+1}=\boldsymbol{m}_{i}+\boldsymbol{C} \mathbf{v}_{i}$

$-\forall p \in \bullet \llbracket \mathbf{v}_{i} \rrbracket^{\bullet} \boldsymbol{m}_{i}[p]>0$ and $\boldsymbol{m}_{i+1}[p]>0$.

Applying lemma 12 , for all $i \geq 1$ there exists $\sigma_{i}$ such that $\boldsymbol{m}_{i} \stackrel{\sigma_{i}}{\longrightarrow} \boldsymbol{m}_{i+1}$. Since $\lim _{i \rightarrow \infty} \boldsymbol{m}_{i}=\boldsymbol{m}$, the sequence $\sigma=\sigma_{1} \sigma_{2} \ldots$ is the required sequence.

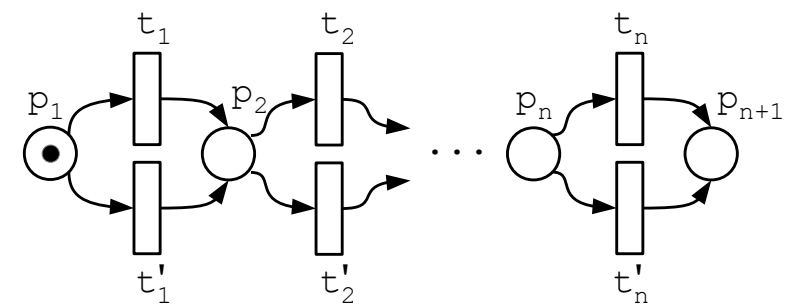

Fig. 3. a CPN system with an exponentially sized firing set.

The key concept in order to get characterisation of properties, is the notion of firing set of a CPN system [9].

Definition 14 Let $\left\langle\mathcal{N}, \boldsymbol{m}_{0}\right\rangle$ be a $C P N$ system. Then its firing set $F S\left(\mathcal{N}, \boldsymbol{m}_{0}\right) \subseteq$ $2^{T}$ is defined by:

$$
F S\left(\mathcal{N}, \boldsymbol{m}_{0}\right)=\left\{\llbracket \vec{\sigma} \rrbracket \mid \boldsymbol{m}_{0} \stackrel{\sigma}{\longrightarrow}\right\}
$$

Due to the empty sequence, $\emptyset \in F S\left(\mathcal{N}, \boldsymbol{m}_{0}\right)$. The size of a firing set may be exponential w.r.t. the number of transitions of the net. For example, consider the CPN system of Fig. 3. Its firing set is:

$$
\left\{T^{\prime} \mid \forall 1 \leq j<i \leq n\left\{t_{i}, t_{i}^{\prime}\right\} \cap T^{\prime} \neq \emptyset \Rightarrow\left\{t_{j}, t_{j}^{\prime}\right\} \neq \emptyset\right\}
$$

Thus its size is at least $2^{\frac{|T|}{2}}$.

The next two lemmas establish elementary properties of the firing set and leads to new notions.

Lemma 15 Let $\mathcal{N}$ be a $C P N$ and $\boldsymbol{m}, \boldsymbol{m}^{\prime}$ be two markings such that $\llbracket \boldsymbol{m} \rrbracket=\llbracket \boldsymbol{m}^{\prime} \rrbracket$. Then $F S(\mathcal{N}, \boldsymbol{m})=F S\left(\mathcal{N}, \boldsymbol{m}^{\prime}\right)$. 
Proof. Since $\llbracket \boldsymbol{m} \rrbracket=\llbracket \boldsymbol{m}^{\prime} \rrbracket$, there exists $\alpha>0$ such that $\alpha \boldsymbol{m} \leq \boldsymbol{m}^{\prime}$. Let $\boldsymbol{m} \stackrel{\sigma}{\longrightarrow}$. Using lemma $11 \alpha \boldsymbol{m} \stackrel{\alpha \sigma}{\longrightarrow}$. Since $\alpha \boldsymbol{m} \leq \boldsymbol{m}^{\prime}, \boldsymbol{m}^{\prime} \stackrel{\alpha \sigma}{\longrightarrow}$. Thus $F S(\mathcal{N}, \boldsymbol{m}) \subseteq F S\left(\mathcal{N}, \boldsymbol{m}^{\prime}\right)$. By symmetry, $F S(\mathcal{N}, \boldsymbol{m})=F S\left(\mathcal{N}, \boldsymbol{m}^{\prime}\right)$.

So given $P^{\prime} \subseteq P$, without ambiguity we define $F S\left(\mathcal{N}, P^{\prime}\right)$ by:

$$
F S\left(\mathcal{N}, P^{\prime}\right) \stackrel{\text { def }}{=} F S(\mathcal{N}, \boldsymbol{m}) \text { for any } \boldsymbol{m} \text { such that } P^{\prime}=\llbracket \boldsymbol{m} \rrbracket
$$

Lemma 16 Let $\left\langle\mathcal{N}, \boldsymbol{m}_{0}\right\rangle$ be a CPN system. Then $F S\left(\mathcal{N}, \boldsymbol{m}_{0}\right)$ is closed by union.

Proof. Let $\boldsymbol{m}_{0} \stackrel{\sigma}{\longrightarrow}$ and $\boldsymbol{m}_{0} \stackrel{\sigma^{\prime}}{\longrightarrow}$.

Then using three times lemma $11,0.5 \boldsymbol{m}_{0} \stackrel{0.5 \sigma}{\longrightarrow}, 0.5 \boldsymbol{m}_{0} \stackrel{0.5 \sigma^{\prime}}{\longrightarrow}$ and $\boldsymbol{m}_{0} \stackrel{0.5 \sigma+0.5 \sigma^{\prime}}{\longrightarrow}$. Since $\llbracket \overrightarrow{0.5 \sigma+0.5 \sigma^{\prime}} \rrbracket=\llbracket \vec{\sigma} \rrbracket \cup \llbracket \overrightarrow{\sigma^{\prime}} \rrbracket$, the conclusion follows.

Notation. We denote $\operatorname{maxFS}\left(\mathcal{N}, \boldsymbol{m}_{0}\right)$ the maximal set of $F S\left(\mathcal{N}, \boldsymbol{m}_{0}\right)$ that is the union of all members of $F S\left(\mathcal{N}, \boldsymbol{m}_{0}\right)$.

The next proposition is a structural characterisation for a subset of transitions to belong to the firing set. In addition, it shows that in the positive case, a "useful" corresponding sequence always exists and furthermore one may build this sequence in polynomial time.

Proposition 17 Let $\left\langle\mathcal{N}, \boldsymbol{m}_{0}\right\rangle$ be a $C P N$ system and $T^{\prime}$ be a subset of transitions. Then:

$$
T^{\prime} \in F S\left(\mathcal{N}, \boldsymbol{m}_{0}\right) \text { iff } \mathcal{N}_{T^{\prime}} \text { has no empty siphon in } \boldsymbol{m}_{0} .
$$

Furthermore if $T^{\prime} \in F S\left(\mathcal{N}, \boldsymbol{m}_{0}\right)$ then there exists $\sigma=\alpha_{1} t_{1} \ldots \alpha_{k} t_{k}$ with $\alpha_{i}>0$ for all $i, T^{\prime}=\left\{t_{1}, \ldots, t_{k}\right\}$ and a marking $\boldsymbol{m}$ such that:

$-\boldsymbol{m}_{0} \stackrel{\sigma}{\longrightarrow} \boldsymbol{m}$

- for all place $p, \boldsymbol{m}(p)>0$ iff $\boldsymbol{m}_{0}(p)>0$ or $p \in{ }^{\bullet} T^{\bullet}$.

Proof.

Necessity. Suppose $\mathcal{N}_{T^{\prime}}$ contains an empty siphon $\Sigma$ in $\boldsymbol{m}_{0}$. Then none of the transitions belonging $\Sigma^{\bullet}$ can be fired in the future. Since $\mathcal{N}_{T^{\prime}}$ does not contain isolated places $\Sigma^{\bullet}\left(=\Sigma^{\bullet}\right) \neq \emptyset$ and so $T^{\prime} \notin F S\left(\mathcal{N}, \boldsymbol{m}_{0}\right)$.

Sufficiency. Suppose that $\mathcal{N}_{T^{\prime}}$ has no empty siphon in $\boldsymbol{m}_{0}$. We build by induction the sequence $\sigma$ of the proposition. More precisely, we inductively prove for increasing values of $i$ that:

- for every $j<i$ there exists a non empty set of transitions $T_{j} \subseteq T^{\prime}$ that fulfill for all $j \neq j^{\prime}, T_{j} \cap T_{j^{\prime}}=\emptyset$;

- for every $j \leq i$ there exists a marking $\boldsymbol{m}_{j}$ with $\boldsymbol{m}_{j}(p)>0$ iff $\boldsymbol{m}_{0}(p)>0$ or $p \in{ }^{\bullet} T_{k}$ ' for some $k<j$;

- for every $j<i$ there exists a sequence $\sigma_{j}=\alpha_{j, 1} t_{j, 1} \ldots \alpha_{j, k_{j}} t_{j, k_{j}}$ with $T_{j}=\left\{t_{j, 1} \ldots t_{j, k_{j}}\right\}$ and $\boldsymbol{m}_{j} \stackrel{\sigma}{\longrightarrow} \boldsymbol{m}_{j+1}$.

There is nothing to prove for the basis case $i=0$.

Suppose that the assertion holds until $i$. If $T^{\prime}=T_{1} \cup \ldots \cup T_{i-1}$ then we are done. Otherwise define $T^{\prime \prime}=T^{\prime} \backslash\left(T_{1} \cup \ldots \cup T_{i-1}\right)$ and $T_{i}=\left\{t\right.$ enabled in $\boldsymbol{m}_{i} \mid t \in$ $\left.T^{\prime \prime}\right\}$. We claim that $T_{i}$ is not empty. Otherwise for all $t \in T^{\prime \prime}$, there exists 
an empty place $p_{t}$ in $\boldsymbol{m}_{i}$. Due to the inductive hypothesis, $\boldsymbol{m}_{0}\left(p_{t}\right)=0$ and - $p_{t} \cap\left(T_{1} \cup \ldots \cup T_{i-1}\right)=\emptyset$. So the union of places $p_{t}$ is an empty siphon of $\left\langle\mathcal{N}_{T^{\prime}}, \boldsymbol{m}_{0}\right\rangle$ which contradicts our hypothesis.

Let us denote $T_{i}=\left\{t_{i, 1} \ldots t_{i, k_{i}}\right\}$. Define $\alpha=\min \left(\frac{m_{i}(p)}{2 k_{i}} \mid p \in{ }^{\bullet} T_{i}\right)$ with the convention that $\alpha=1$ if $\bullet T_{i}=\emptyset$. The sequence $\sigma_{i}=\alpha t_{i, 1} \ldots \alpha t_{i, k_{i}}$ is fireable from $\boldsymbol{m}_{i}$ and leads to a marking $\boldsymbol{m}_{i+1}$ fulfilling the inductive hypothesis.

Since $T^{\prime \prime}$ is finite the procedure terminates.

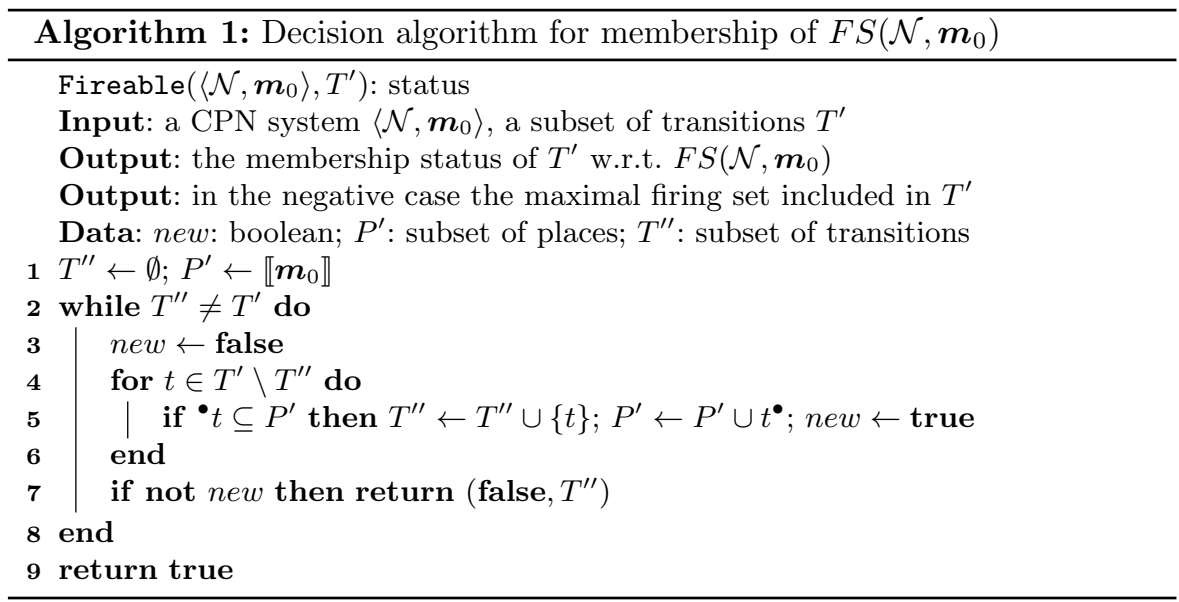

We include the complexity result below since its proof relies in a straightforward manner on the sufficiency proof of the previous proposition.

Corollary 18 Let $\left\langle\mathcal{N}, \boldsymbol{m}_{0}\right\rangle$ be a CPN system and $T^{\prime}$ be a subset of transitions. Then algorithm 1 checks in polynomial time whether $T^{\prime} \in F S\left(\mathcal{N}, \boldsymbol{m}_{0}\right)$ and in the negative case returns the maximal firing set included in $T^{\prime}$ (when called with $T=T^{\prime}$, it returns $\left.\operatorname{maxFS}\left(\mathcal{N}, \boldsymbol{m}_{0}\right)\right)$.

\subsection{Characterisation of reachability and boundedness}

In [9] a characterisation of reachability was presented. The theorem below is an alternative characterisation that only relies on the state equation and firing sets.

Theorem 19 Let $\left\langle\mathcal{N}, \boldsymbol{m}_{0}\right\rangle$ be a CPN system and $\boldsymbol{m}$ be a marking.

Then $\boldsymbol{m} \in \operatorname{RS}\left(\mathcal{N}, \boldsymbol{m}_{0}\right)$ iff there exists $\mathbf{v} \in \mathbb{R}_{\geq 0}^{|T|}$ such that:

1. $m=m_{0}+C \mathbf{v}$

2. $\llbracket \mathbf{v} \rrbracket \in F S\left(\mathcal{N}, \boldsymbol{m}_{0}\right)$

3. $\llbracket \mathbf{v} \rrbracket \in F S\left(\mathcal{N}^{-1}, \boldsymbol{m}\right)$ 


\section{Proof.}

Necessity. Let $\boldsymbol{m} \in \operatorname{RS}\left(\mathcal{N}, \boldsymbol{m}_{0}\right)$. So there exists a finite firing sequence $\sigma$ such that $\boldsymbol{m}_{0} \stackrel{\sigma}{\longrightarrow} \boldsymbol{m}$. Let $\mathbf{v}=\vec{\sigma}$, then $\boldsymbol{m}=\boldsymbol{m}_{0}+\boldsymbol{C} \mathbf{v}$.

Since $\sigma$ is fireable from $\boldsymbol{m}_{o}$ in $\mathcal{N}, \llbracket \mathbf{v} \rrbracket \in F S\left(\mathcal{N}, \boldsymbol{m}_{0}\right)$. In $\mathcal{N}^{-1}, \boldsymbol{m} \stackrel{\sigma^{-1}}{\longrightarrow} \boldsymbol{m}_{0}$. Since $\mathbf{v}=\overrightarrow{\sigma^{-1}}, \llbracket \mathbf{v} \rrbracket \in F S\left(\mathcal{N}^{-1}, \boldsymbol{m}\right)$.

Sufficiency. Since $\llbracket \mathbf{v} \rrbracket \in F S\left(\mathcal{N}, \boldsymbol{m}_{0}\right)$, using Proposition 17 and Lemma 11 there exists a sequence $\sigma_{1}$ such that $\llbracket \mathbf{v} \rrbracket=\llbracket \overrightarrow{\sigma_{1}} \rrbracket$, for all $0<\alpha_{1} \leq 1, \boldsymbol{m}_{0} \stackrel{\alpha_{1} \sigma_{1}}{\longrightarrow} \boldsymbol{m}_{1}$ with $\boldsymbol{m}_{1}(p)>0$ for $p \in \in^{\bullet} \llbracket \mathbf{v} \rrbracket^{\bullet}$.

Since $\llbracket \mathbf{v} \rrbracket \in F S\left(\mathcal{N}^{-1}, \boldsymbol{m}\right)$, using Proposition 17 and Lemma 11 there exists a sequence $\sigma_{2}$ such that $\llbracket \mathbf{v} \rrbracket=\llbracket \overrightarrow{\sigma_{2}} \rrbracket$, for all $0<\alpha_{2} \leq 1, \boldsymbol{m} \stackrel{\alpha_{2} \sigma_{2}}{\longrightarrow} \boldsymbol{m}_{2}$ in $\mathcal{N}^{-1}$ with $\boldsymbol{m}_{2}(p)>0$ for $p \in \bullet \llbracket \mathbf{v} \rrbracket$.

Choose $\alpha_{1}$ and $\alpha_{2}$ enough small such that the vector $\mathbf{v}^{\prime}=\mathbf{v}-\alpha_{1} \overrightarrow{\sigma_{1}}-\alpha_{2} \overrightarrow{\sigma_{2}}$ is non negative and $\llbracket \mathbf{v}^{\prime} \rrbracket=\llbracket \mathbf{v} \rrbracket$. This is possible since $\llbracket \mathbf{v} \rrbracket=\llbracket \overrightarrow{\sigma_{1}} \rrbracket=\llbracket \overrightarrow{\sigma_{2}} \rrbracket$.

Since $\boldsymbol{m}_{2}=\boldsymbol{m}_{1}+\boldsymbol{C} \mathbf{v}^{\prime}$ and $\boldsymbol{m}_{1}, \boldsymbol{m}_{2}$ fulfill the hypotheses of Lemma 12, there exists a sequence $\sigma_{3}$ such that $\mathbf{v}^{\prime}=\overrightarrow{\sigma_{3}}$ and $\boldsymbol{m}_{1} \stackrel{\sigma_{3}}{\longrightarrow} \boldsymbol{m}_{2}$.

Let $\sigma=\left(\alpha_{1} \sigma_{1}\right) \sigma_{3}\left(\alpha_{2} \sigma_{2}\right)^{-1}$ then $\boldsymbol{m}_{0} \stackrel{\sigma}{\longrightarrow} \boldsymbol{m}$.

The following characterisation has been stated in [9]. We include the proof here since in that paper, the proof of necessity was not developed.

Theorem 20 Let $\left\langle\mathcal{N}, \boldsymbol{m}_{0}\right\rangle$ be a CPN system and $\boldsymbol{m}$ be a marking.

Then $\boldsymbol{m} \in \lim -\operatorname{RS}\left(\mathcal{N}, \boldsymbol{m}_{0}\right)$ iff there exists $\mathbf{v} \in \mathbb{R}_{\geq 0}^{|T|}$ such that:

1. $\boldsymbol{m}=\boldsymbol{m}_{0}+C \mathbf{v}$

2. $\llbracket \mathbf{v} \rrbracket \in F S\left(\mathcal{N}, \boldsymbol{m}_{0}\right)$

Proof.

Necessity. Let $\boldsymbol{m} \in \lim -\operatorname{RS}\left(\mathcal{N}, \boldsymbol{m}_{0}\right)$. So there exists a firing sequence $\sigma=$ $\alpha_{1} t_{1} \ldots \alpha_{n} t_{n} \ldots$ such that $\boldsymbol{m}=\lim _{n \rightarrow \infty} \boldsymbol{m}_{n}$, where $\boldsymbol{m}_{n} \stackrel{\alpha_{n+1} t_{n+1}}{\longrightarrow} \boldsymbol{m}_{n+1}$.

Thus there exists $B \in \mathbb{N}$ such that for all $p \in P$ and all $n \in \mathbb{N}, \boldsymbol{m}_{n}[p] \leq B$.

Let $T^{\prime} \stackrel{\text { def }}{=}\left\{t \mid \exists i \in \mathbb{N} t=t_{i}\right\}$. There exists $n_{0}$ such that $T^{\prime}=\left\{t \mid \exists i \leq n_{0} t=t_{i}\right\}$ and so $T^{\prime} \in F S\left(\mathcal{N}, \boldsymbol{m}_{0}\right)$.

Let $\alpha \in \mathbb{Q}_{>0}$ such that $\alpha \leq \min \left(\sum_{i \leq n_{0}, t_{i}=t} \alpha_{i} \mid t \in T^{\prime}\right)$.

Let us define $L P_{n}$ an existential linear program where $\mathbf{v} \in \mathbb{R}^{T}$ is the vector of variables by:

1. $\boldsymbol{m}_{n}-\boldsymbol{m}_{0}=\mathbf{C v}$

2. $\forall t \in T^{\prime} \mathbf{v}[t] \geq \alpha$

3. $\forall t \in T \backslash T^{\prime} \mathbf{v}[t]=0$

Due to the existence of the firing sequence $\sigma$, for all $n \geq n_{0} L P_{n}$ admits a solution. Using linear programming theory (see [11]), since $\boldsymbol{m}_{n}[p] \leq B$ for all $\mathrm{n}$ and all $p$, there exists $B^{\prime}$ such that for all $n \geq n_{0}, L P_{n}$ admits a solution $\mathbf{v}_{n}$ whose items are bounded by $B^{\prime}$. 
So the sequence $\left\{\mathbf{v}_{n}\right\}_{n \geq n_{0}}$ admits a subsequence that converges to some $\mathbf{v}$. By continuity, $\mathbf{v}$ fulfills $\boldsymbol{m}-\boldsymbol{m}_{0}=\boldsymbol{C} \mathbf{v}, \forall t \in T^{\prime} \mathbf{v}[t] \geq \alpha$ and $\forall t \in T \backslash T^{\prime} \mathbf{v}[t]=0$. So $\llbracket \mathbf{v} \rrbracket=T^{\prime}$ and $\mathbf{v}$ is the desired vector.

Sufficiency. Since $\llbracket \mathbf{v} \rrbracket \in F S\left(\mathcal{N}, \boldsymbol{m}_{0}\right)$, using Proposition 17 and Lemma 11 there exists a sequence $\sigma_{1}$ such that $\llbracket \mathbf{v} \rrbracket=\llbracket \overrightarrow{\sigma_{1}} \rrbracket$, for all $0<\alpha_{1} \leq 1, \boldsymbol{m}_{0} \stackrel{\alpha_{1} \sigma_{1}}{\longrightarrow} \boldsymbol{m}_{1}$ with $\boldsymbol{m}_{1}(p)>0$ for $p \in \bullet \llbracket \mathbf{v} \rrbracket^{\bullet}$.

Choose $\alpha_{1}$ enough small such that the vector $\mathbf{v}^{\prime}=\mathbf{v}-\alpha_{1} \overrightarrow{\sigma_{1}}$ is non negative and $\llbracket \mathbf{v}^{\prime} \rrbracket=\llbracket \mathbf{v} \rrbracket$. This is possible since $\llbracket \mathbf{v} \rrbracket=\llbracket \overrightarrow{\sigma_{1}} \rrbracket$.

Since $\boldsymbol{m}=\boldsymbol{m}_{1}+\boldsymbol{C v}^{\prime}$ and $\boldsymbol{m}_{1}$ fulfills the hypotheses of lemma 13, there exists an infinite sequence $\sigma_{2}$ such that $\mathbf{v}^{\prime}=\overrightarrow{\sigma_{2}}$ and $\boldsymbol{m}_{1} \stackrel{\sigma_{2}}{\longrightarrow} \boldsymbol{m}$.

Let $\sigma=\left(\alpha_{1} \sigma_{1}\right) \sigma_{2}$ then $\boldsymbol{m}_{0} \stackrel{\sigma}{\longrightarrow} \boldsymbol{m}$.

We present below the first characterisation of boundedness for CPN systems.

Theorem 21 Given a CPN system $\left\langle\mathcal{N}, \boldsymbol{m}_{0}\right\rangle$. Then $\left\langle\mathcal{N}, \boldsymbol{m}_{0}\right\rangle$ is unbounded iff: There exists $\mathbf{v} \in \mathbb{R}_{\geq 0}^{T}$ such that $\boldsymbol{C} \mathbf{v} \gtrless \mathbf{0}$ and $\llbracket \mathbf{v} \rrbracket \subseteq \operatorname{maxFS}\left(\mathcal{N}, \boldsymbol{m}_{0}\right)$.

Proof.

Sufficiency. Assume there exists $\mathbf{v} \in \mathbb{R}_{\geq 0}^{T}$ such that $\boldsymbol{C} \mathbf{v} \gtrless \mathbf{0}$ and $\llbracket \mathbf{v} \rrbracket \subseteq$ $\operatorname{maxFS}\left(\mathcal{N}, \boldsymbol{m}_{0}\right)$. Denote $T^{\prime} \stackrel{\text { def }}{=} \operatorname{maxFS}\left(\mathcal{N}, \boldsymbol{m}_{0}\right)$. Using proposition 17 , there exists $\boldsymbol{m}_{1} \in R S\left(\mathcal{N}, \boldsymbol{m}_{0}\right)$ such that for all $p \in{ }^{\bullet} T^{\prime}{ }^{\bullet}, \boldsymbol{m}_{1}(p)>0$. Define $\boldsymbol{m}_{2} \stackrel{\text { def }}{=} \boldsymbol{m}_{1}+\boldsymbol{C} \mathbf{v}$, thus $\boldsymbol{m}_{2} \gtrless \boldsymbol{m}_{1}$. Since $\llbracket \mathbf{v} \rrbracket \subseteq T^{\prime}, \boldsymbol{m}_{1}$ and $\boldsymbol{m}_{2}$ fulfill the hypotheses of lemma 12 . Applying it, yields a firing sequence $\boldsymbol{m}_{1} \stackrel{\sigma}{\longrightarrow} \boldsymbol{m}_{2}$. Iterating this sequence establishes the unboundedness of $\left\langle\mathcal{N}, \boldsymbol{m}_{0}\right\rangle$.

Necessity. Assume $\left\langle\mathcal{N}, \boldsymbol{m}_{0}\right\rangle$ is unbounded. Then there exists $p \in P$ and a family of firing sequences $\left\{\sigma_{n}\right\}_{n \in \mathbb{N}}$ such that $\boldsymbol{m}_{0} \stackrel{\sigma_{n}}{\longrightarrow} \boldsymbol{m}_{n}$ and $\boldsymbol{m}_{n}(p) \geq n$. Since $\left\{\llbracket \vec{\sigma}_{n} \rrbracket\right\}_{n \in \mathcal{N}}$ is finite by extracting a subsequence w.l.o.g. we can assume that all these sequences have the same support, say $T^{\prime} \subseteq \operatorname{maxFS}\left(\mathcal{N}, \boldsymbol{m}_{0}\right)$.

Let $\mathbf{v}_{n} \stackrel{\text { def }}{=} \boldsymbol{C} \vec{\sigma}_{n}$. Define $\mathbf{w}_{n}=\frac{\mathbf{v}_{n}}{\left\|\mathbf{v}_{n}\right\|_{1}}$. Since $\left\{\mathbf{w}_{n}\right\}_{n \in \mathbb{N}}$ belongs to a compact set, there exists a convergent subsequence $\left\{\mathbf{w}_{\alpha(n)}\right\}_{n \in \mathbb{N}}$. Denote $\mathbf{w}$ its limit. Since $\|\mathbf{w}\|_{1}=1, \mathbf{w}$ is non null. We claim that $\mathbf{w}$ is a non negative vector. Since $\boldsymbol{m}_{n}(p) \geq n,\left\|\mathbf{v}_{n}\right\|_{1} \geq \mathbf{v}_{n}[p] \geq n-\boldsymbol{m}_{0}[p]$. On the other hand, for all $p^{\prime} \in P$, $\mathbf{w}_{n}\left[p^{\prime}\right] \geq \frac{-\boldsymbol{m}_{0}\left[p^{\prime}\right]}{\left\|\mathbf{v}_{n}\right\|_{1}}$. Combining the two inequalities, for $n>\boldsymbol{m}_{0}[p], \mathbf{w}_{n}\left[p^{\prime}\right] \geq$

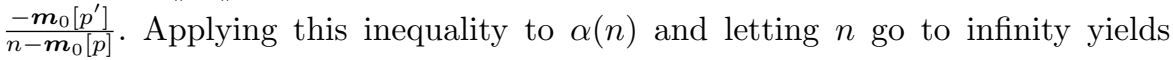
$\mathbf{w}\left[p^{\prime}\right] \geq 0$.

Due to standard results of polyhedra theory (see [1] for instance), the set

$\left\{\boldsymbol{C}_{P \times T^{\prime}} \mathbf{u} \mid \mathbf{u} \in \mathbb{R}_{\geq 0}^{T^{\prime}}\right\}$ is closed. So there exists $\mathbf{u} \in \mathbb{R}_{\geq 0}^{T^{\prime}}$ such that $\mathbf{w}=\boldsymbol{C u}$. Considering $\mathbf{u}$ as a vector of $\mathbb{R}_{\geq 0}^{T}$ by adding null components for $T \backslash T^{\prime}$ yields the required vector.

\section{Decision procedures}

Naively implementing the characterisation of reachability would lead to an exponential procedure since it would require to enumerate the items of $F S\left(\mathcal{N}, \boldsymbol{m}_{0}\right)$ 
(whose size is possibly exponential). For each item, say $T^{\prime}$, the algorithm would check in polynomial time (1) whether $T^{\prime}$ belongs to $F S\left(\mathcal{N}^{-1}, \boldsymbol{m}\right)$ and $(2)$ whether the associated linear program $\mathbf{v}>\mathbf{0} \wedge \boldsymbol{C}_{P \times T^{\prime}} \mathbf{v}=\boldsymbol{m}-\boldsymbol{m}_{0}$ admits a solution. Guessing $T^{\prime}$ shows that the reachability problem belongs to NP.

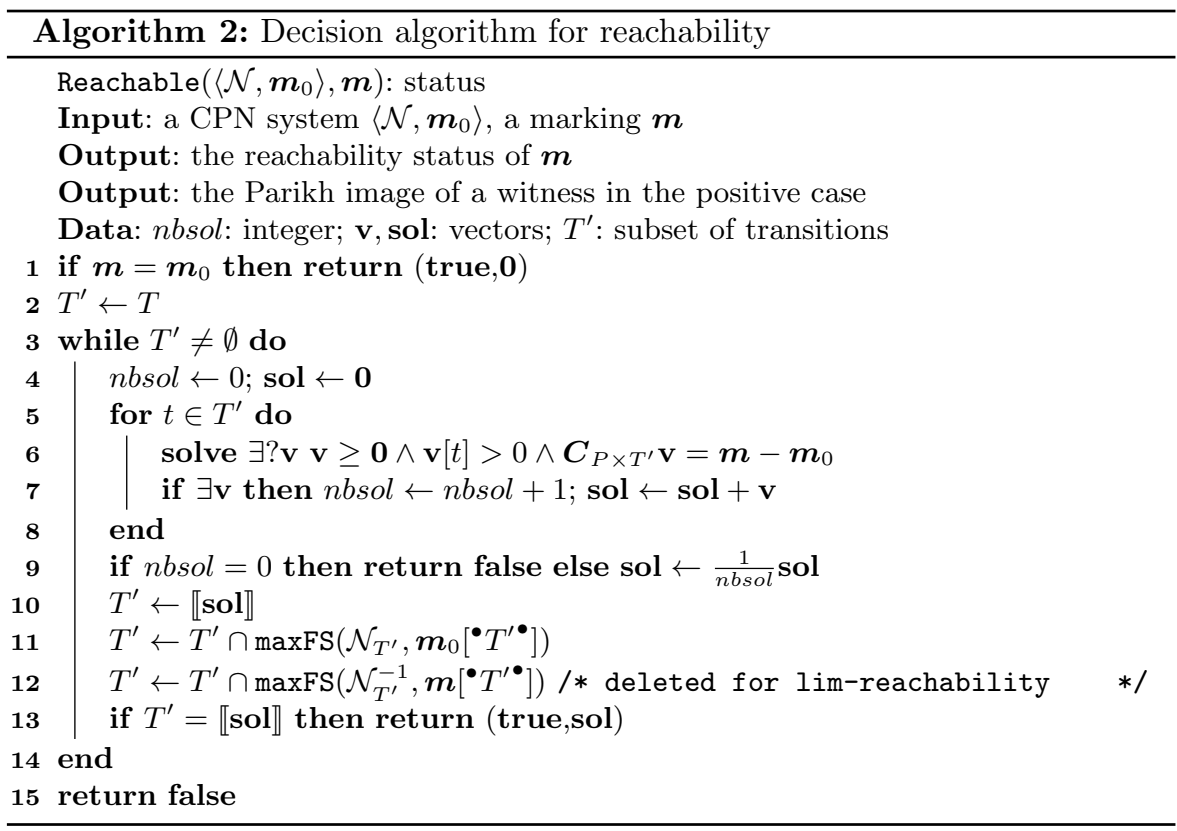

In fact, we improve this upper bound with the help of Algorithm 2. When $\boldsymbol{m} \neq \boldsymbol{m}_{0}$, this algorithm maintains a subset of transitions $T^{\prime}$ which fulfills $\llbracket \vec{\sigma} \rrbracket \subseteq T^{\prime}$ for any $\boldsymbol{m}_{0} \stackrel{\sigma}{\longrightarrow} \boldsymbol{m}$ (as will be proven in proposition 22 ). Initially $T^{\prime}$ is set to $T$. Then lines 4-9 build a solution to the state equation restricted to transitions of $T^{\prime}$ with a maximal support (if there is at least one). If there is no solution then the algorithm returns false. Otherwise $T^{\prime}$ is successively restricted to (1) the support of this maximal solution (line 10), (2) the maximal firing set in $\operatorname{maxFS}\left(\mathcal{N}_{T^{\prime}}, \boldsymbol{m}_{0}\left[\boldsymbol{\bullet}^{\prime}\right]\right.$ ) (line 11) and, (3) the maximal firing set in $\operatorname{maxFS}\left(\mathcal{N}_{T^{\prime}}^{-1}, \boldsymbol{m}\left[T^{\bullet} T^{\prime \bullet}\right)\right.$ (line 12). If the two last restrictions do not modify $T^{\prime}$ then the algorithm returns true. If $T^{\prime}$ becomes empty then the algorithm returns false.

Omitting line 12, Algorithm 2 decides the lim-reachability problem.

Proposition 22 Algorithm 2 returns true iff $\boldsymbol{m}$ is reachable in $\left\langle\mathcal{N}, \boldsymbol{m}_{0}\right\rangle$. Algorithm 2 without line 12 returns true iff $\boldsymbol{m}$ is lim-reachable in $\left\langle\mathcal{N}, \boldsymbol{m}_{0}\right\rangle$.

Proof. We only consider the non trivial case $\boldsymbol{m} \neq \boldsymbol{m}_{0}$.

Soundness. Assume that the algorithm returns true at line 13 .

By definition, vector sol which is a barycenter of solutions is also a solution 
with maximal support and so fulfils the first statement of Theorem 19. Since $T^{\prime}=\llbracket \mathbf{s o l} \rrbracket$ at line $13, \llbracket \mathbf{s o l} \rrbracket \in F S\left(\mathcal{N}, \boldsymbol{m}_{0}\right)$ due to line 11 and $\llbracket \mathbf{s o l} \rrbracket \in F S\left(\mathcal{N}^{-1}, \boldsymbol{m}\right)$ due to line 12 . Thus $\boldsymbol{m}$ is reachable in $\left\langle\mathcal{N}, \boldsymbol{m}_{0}\right\rangle$ since it fulfills the assertions of Theorem 19. In case of lim-reachability, line 12 is omitted. So the assertions of Theorem 20 are fulfilled and $\boldsymbol{m}$ is lim-reachable in $\left\langle\mathcal{N}, \boldsymbol{m}_{0}\right\rangle$.

Completeness. Assume the algorithm returns false.

We claim that at any time the algorithm fulfils the following invariant: for any $\boldsymbol{m}_{0} \stackrel{\sigma}{\longrightarrow} \boldsymbol{m}, \llbracket \vec{\sigma} \rrbracket \subseteq T^{\prime}$.

This invariant initially holds since $T^{\prime}=T$. At line 10 due to the first assertion of Theorem 19, for any such $\sigma$, $\vec{\sigma} \rrbracket \subseteq \llbracket$ sol $\rrbracket$ since sol is a solution with maximal support. So the assignment of line 10 lets true the invariant. Due to the second assertion of Theorem 19 and the invariant, any $\sigma$ fulfils $\llbracket \vec{\sigma} \rrbracket \subseteq \operatorname{maxFS}\left(\mathcal{N}_{T^{\prime}}, \boldsymbol{m}_{0}\left[{ }^{\bullet} T^{\prime \bullet}\right]\right)$. So the assignment of line 11 lets true the invariant. Due to the third assertion of Theorem 19 and the invariant, any $\sigma$ fulfils $\llbracket \vec{\sigma} \rrbracket \subseteq \operatorname{maxFS}\left(\mathcal{N}_{T^{\prime}}^{-1}, \boldsymbol{m}\left[{ }^{\bullet} T^{\prime \bullet}\right]\right)$. So the assignment of line 12 lets true the invariant. If the algorithm returns false at line 9 due to the invariant the first assertion of Theorem 19 cannot be satisfied. If the algorithm returns false at line 15 then $T^{\prime}=\emptyset$. So due to the invariant and since $\boldsymbol{m} \neq \boldsymbol{m}_{0}, \boldsymbol{m}$ is not reachable from $\boldsymbol{m}_{0}$. The case of lim-reachability is similarly handled with the following invariant: for any $\boldsymbol{m}_{0} \stackrel{\sigma}{\longrightarrow} \boldsymbol{m}, \llbracket \vec{\sigma} \rrbracket \subseteq T^{\prime}$.

Proposition 23 The reachability and the lim-reachability problems for CPN systems are decidable in polynomial time.

Proof. Let us analyse the time complexity of Algorithm 2. Since $T^{\prime}$ must be modified in lines 11 or 12 in order to start a new iteration of the main loop, there are at most $|T|$ iterations of this loop. The number of iterations of the inner loop is also bounded by $|T|$. Finally solving a linear program can be performed in polynomial time [11] as well as computing the maximal item of a firing set (see corollary 18).

In [9], it is proven that the lim-reachability problem for consistent CPN systems with no empty siphons in the initial marking is decidable in polynomial time. We improve this result by showing that this problem and a similar one belong to $\mathrm{NC} \subseteq$ PTIME (a complexity class of problems that can take advantage of parallel computations, see [10]).

Proposition 24 The reachability problem for consistent CPN systems with no empty siphons in the initial marking and no empty siphons in the final marking for the reverse net belongs to $N C$.

The lim-reachability problem for consistent CPN systems with no empty siphons in the initial marking belongs to $\mathrm{NC}$.

Proof. Due to the assumptions on siphons and proposition 17 only the first assertion of Theorems 19 and 20 needs to be checked. Due to consistency, there exists w $>\mathbf{0}$ such that $\boldsymbol{C} \mathbf{w}=\mathbf{0}$. Assume there is some $\mathbf{v} \in \mathbb{R}^{T}$ such that $\boldsymbol{m}-\boldsymbol{m}_{0}=\boldsymbol{C} \mathbf{v}$. For some $n \in \mathbb{N}$ large enough, $\mathbf{v}^{\prime} \stackrel{\text { def }}{=} \mathbf{v}+n \mathbf{w} \in \mathbb{R}_{\geq 0}^{T}$ and still fulfils $\boldsymbol{m}-\boldsymbol{m}_{0}=C \mathbf{v}^{\prime}$. 
Now the decision problem $\exists$ ? $\mathbf{v} \in \mathbb{R}^{T} \boldsymbol{m}-\boldsymbol{m}_{0}=\boldsymbol{C} \mathbf{v}$ belongs to NC [4].

Proposition 25 The boundedness problem for CPN systems is decidable in polynomial time.

Proof. Using the characterisation of Theorem 21, one first computes in polynomial time $T^{\prime}=\operatorname{maxFS}\left(\mathcal{N}, \boldsymbol{m}_{0}\right)$ (see corollary 18). Then for all $p \in P$, one solves the existential linear program $\exists$ ? $\mathbf{v} \geq \mathbf{0} \boldsymbol{C}_{P \times T^{\prime}} \mathbf{v} \geq \mathbf{0} \wedge\left(\boldsymbol{C}_{P \times T^{\prime}} \mathbf{v}\right)[p]>0$. The $\mathrm{CPN}$ system is unbounded if some of these linear programs admits a solution.

In discrete Petri nets, the reachability set inclusion problem is undecidable, while the restricted problem of home state is decidable (see [7] for a detailed survey about decidability results in PNs). In CPN systems, this problem is decidable thanks to the special structure of the (lim-)reachability sets.

Proposition 26 The reachability set inclusion and the lim-reachability set inclusion problems for CPN systems are decidable in exponential time.

Proof. Let us define $T P \stackrel{\text { def }}{=}\left\{\left(T^{\prime}, P^{\prime}\right) \mid T^{\prime} \in F S\left(\mathcal{N}, \boldsymbol{m}_{0}\right) \wedge P^{\prime} \subseteq P \wedge T^{\prime} \in\right.$ $\left.F S\left(\mathcal{N}^{-1}, P^{\prime}\right)\right\}$. For every pair $\left(T^{\prime}, P^{\prime}\right) \in T P$, define the polyhedron $E_{T^{\prime}, P^{\prime}}$ over $\mathbb{R}^{P} \times \mathbb{R}^{T^{\prime}}$ by:

$$
E_{T^{\prime}, P^{\prime}} \stackrel{\text { def }}{=}\left\{(\boldsymbol{m}, \mathbf{v}) \mid \boldsymbol{m}\left[P^{\prime}\right]>\mathbf{0} \wedge \boldsymbol{m}\left[P \backslash P^{\prime}\right]=\mathbf{0} \wedge \mathbf{v}>\mathbf{0} \wedge \boldsymbol{m}=\boldsymbol{C}_{P \times T^{\prime}} \mathbf{v}\right\}
$$

and $R_{T^{\prime}, P^{\prime}}$ by: $R_{T^{\prime}, P^{\prime}} \stackrel{\text { def }}{=}\left\{\boldsymbol{m} \mid \exists \mathbf{v}(\boldsymbol{m}, \mathbf{v}) \in E_{T^{\prime}, P^{\prime}}\right\}$

Using the characterisation of Theorem 19 and Lemma 15,

$R S\left(\mathcal{N}, \boldsymbol{m}_{0}\right)=\bigcup_{\left(T^{\prime}, P^{\prime}\right) \in T P} R_{T^{\prime}, P^{\prime}}$.

Due to Lemma 11, the reachability set of a CPN system is convex. So $R S\left(\mathcal{N}, \boldsymbol{m}_{0}\right)$ can be rewritten as:

$$
\begin{gathered}
R S\left(\mathcal{N}, \boldsymbol{m}_{0}\right)=\left\{\sum_{\left(T^{\prime}, P^{\prime}\right) \in T P} \lambda_{T^{\prime}, P^{\prime}} \boldsymbol{m}_{T^{\prime}, P^{\prime}} \mid\right. \\
\left.\sum_{\left(T^{\prime}, P^{\prime}\right) \in T P} \lambda_{T^{\prime}, P^{\prime}}=1 \wedge \forall\left(T^{\prime}, P^{\prime}\right) \in T P \lambda_{T^{\prime}, P^{\prime}} \geq 0 \wedge \boldsymbol{m}_{T^{\prime}, P^{\prime}} \in R_{T^{\prime}, P^{\prime}}\right\}
\end{gathered}
$$

Observe that this representation is exponential w.r.t. the size of the CPN system. Let $\left\langle\mathcal{N}, \boldsymbol{m}_{0}\right\rangle$ and $\left\langle\mathcal{N}^{\prime}, \boldsymbol{m}_{0}^{\prime}\right\rangle$ be two CPN systems for which one wants to check whether $R S\left(\mathcal{N}, \boldsymbol{m}_{0}\right) \subseteq R S\left(\mathcal{N}^{\prime}, \boldsymbol{m}_{0}^{\prime}\right)$. One builds the representation above for $R S\left(\mathcal{N}, \boldsymbol{m}_{0}\right)$ and $R S\left(\mathcal{N}^{\prime}, \boldsymbol{m}_{0}^{\prime}\right)$. Then one transforms the representation of the set $R S\left(\mathcal{N}^{\prime}, \boldsymbol{m}_{0}^{\prime}\right)$ as a system of linear constraints. This can be done in polynomial time w.r.t. the original representation [2]. So the number of constraints is still exponential w.r.t. the size of $\left\langle\mathcal{N}^{\prime}, \boldsymbol{m}_{0}^{\prime}\right\rangle$.

Afterwards for every constraint of this new representation, one adds its negation to the representation of $R S\left(\mathcal{N}, \boldsymbol{m}_{0}\right)$ and check for a solution of such a system. $R S\left(\mathcal{N}, \boldsymbol{m}_{0}\right) \nsubseteq R S\left(\mathcal{N}^{\prime}, \boldsymbol{m}_{0}^{\prime}\right)$ iff at least one of these linear programs admits a solution. The overall complexity of this procedure is still exponential w.r.t. the size of the problem. The procedure for lim-reachability set inclusion is similar. 


\section{Hardness results}

We now provide matching lower bounds for almost all problems analysed in the previous sections.

The proof of this proposition is in the appendix.

Proposition $\mathbf{2 7}$ The reachability, lim-reachability and boundedness problems for CPN systems are PTIME-complete.

We want to prove that the lower bounds are robust. To this aim, we recall free-choice CPNs.

Definition $28 A C P N \mathcal{N}$ is free-choice if:

- $\forall p \in P \forall t \in T\{\boldsymbol{P r e}[p, t], \boldsymbol{P o s t}[p, t]\} \subseteq\{0,1\}$;

$-\forall t, t^{\prime} \in T \cdot t \cap \bullet t^{\prime} \neq \emptyset \Rightarrow \bullet t=\bullet t^{\prime}$.

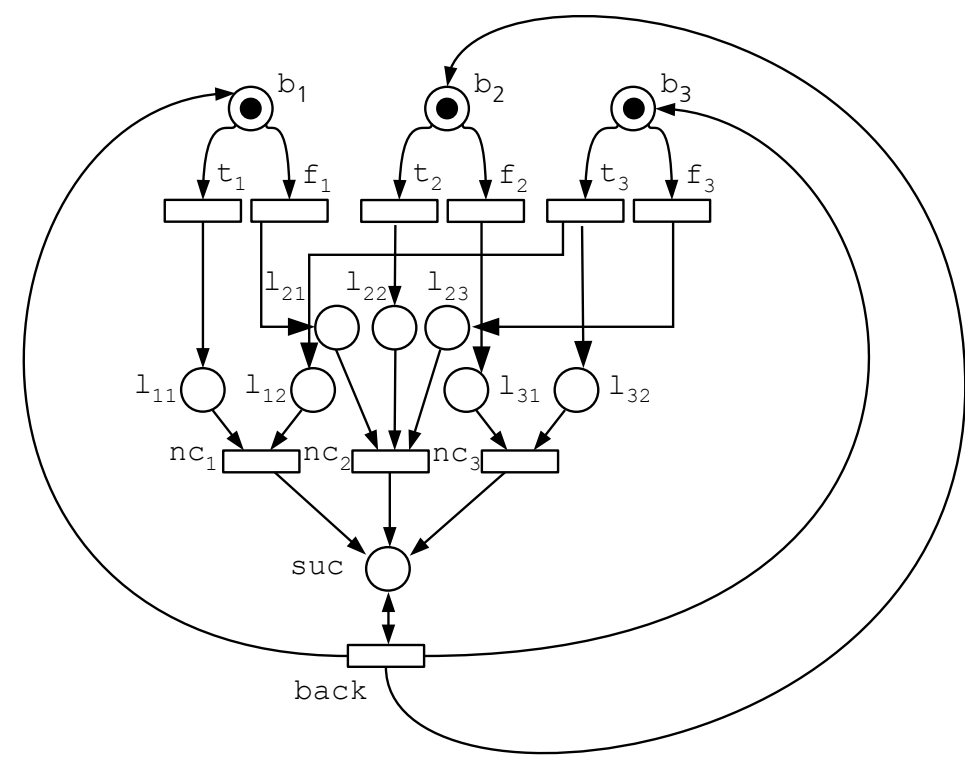

Fig. 4. The CPN corresponding to formula $\left(\neg x_{1} \vee \neg x_{3}\right) \wedge\left(x_{1} \vee \neg x_{2} \vee x_{3}\right) \wedge\left(x_{2} \vee \neg x_{3}\right)$

Proposition 29 The (lim-)deadlock-freeness and (lim-)liveness problems in freechoice CPN systems are coNP-hard. 
Proof. We use almost the same reduction from the 3SAT problem as the one proposed for free-choice Petri nets in [6]. However the proof of correctness is specific to continuous nets.

Let $\left\{x_{1}, x_{2}, \ldots, x_{n}\right\}$ denote the set of propositions and $\left\{c_{1}, c_{2}, \ldots, c_{m}\right\}$ denote the set of clauses. Every clause $c_{j}$ is defined by $c_{j} \stackrel{\text { def }}{=} l i t_{j 1} \vee l i t_{j 2} \vee l i t_{j 3}$ where for all $j, k, l i t_{j k} \in\left\{x_{1}, \ldots, x_{n}, \neg x_{1}, \ldots, \neg x_{n}\right\}$. The satisfiability problem consists in the existence of an interpretation $\nu:\left\{x_{1}, x_{2}, \ldots, x_{n}\right\} \longrightarrow\{$ false, true $\}$, such that for all clause $c_{j}, \nu\left(c_{j}\right)=$ true.

Every proposition $x_{i}$ yields a place $b_{i}$ initially marked with a token (all other places are unmarked) and input of two transitions $t_{i}, f_{i}$ corresponding to the assignment associated with an interpretation. Every of literal $l i t_{j k}$ yields a place $l_{j k}$ which is the output of transition $t_{i}$ if $l i t_{j k}=x_{i}$ or transition $f_{i}$ if $l i t_{j k}=\neg x_{i}$ Every clause $c_{j}$ yields a transition $n c_{j}$ with three input "literal" places corresponding to literals $\neg l i t_{j 1}, \neg l i t_{j 2}, \neg l i t_{j 3}$. An additional place suc is the output of every transition $n c_{j}$. Finally, transition back has suc as a loop place and $b_{i}$ for all $i$ as output places. The reduction is illustrated in Fig. 4.

Assume that there exists $\nu$ such that for all clause $c_{j}, \nu\left(c_{j}\right)=$ true. Then fire the following sequence $\sigma=1 t_{1}^{*} \ldots 1 t_{n}^{*}$ where $t_{i}^{*}=t_{i}$ when $\nu\left(x_{i}\right)=$ true and $t_{i}^{*}=f_{i}$ when $\nu\left(x_{i}\right)=$ false. Consider $\boldsymbol{m}$ the reached marking. Since $\nu\left(c_{j}\right)=$ true, at least one input place of $n c_{j}$ is empty in $\boldsymbol{m}$. Moreover $\boldsymbol{m}(s u c)=\boldsymbol{m}\left(b_{i}\right)=0$ for all $i$. So $\boldsymbol{m}$ is dead.

Assume that there does not exist $\nu$ such that for all clause $c_{j}, \nu\left(c_{j}\right)=$ true. Observe that given a marking $\boldsymbol{m}$ such that $\boldsymbol{m}(s u c)>0$ all transitions will be fireable in the future and suc will never decrease (thus $\boldsymbol{m}(s u c)>0$ for a limreachable marking $\boldsymbol{m}$ as well).

So we only consider reachable marking $\boldsymbol{m}$ such that $\boldsymbol{m}(s u c)=0$, i.e. when no transitions $n c_{j}$ have been fired. Our goal is to prove that from such marking there is a sequence that produces tokens in suc. Examining the remaining transitions, the following invariants hold. For all atomic proposition $x_{i}$, and reachable marking $\boldsymbol{m}$, one has

$$
\begin{gathered}
\forall i \boldsymbol{m}\left[b_{i}\right]+\sum_{l_{j k} \in\left\{x_{i}, \neg x_{i}\right\}} \boldsymbol{m}\left[l_{j k}\right] \geq 1 \\
\forall j, k, j^{\prime}, k^{\prime} l i t_{j k}=l i t_{j^{\prime} k^{\prime}} \Rightarrow \boldsymbol{m}\left[l_{j k}\right]=\boldsymbol{m}\left[l_{j^{\prime} k^{\prime}}\right]
\end{gathered}
$$

If for some $i, \boldsymbol{m}\left[b_{i}\right]>0$, we fire $t_{i}$ in order to empty $b_{i}$. Thus the invariants become:

$$
\begin{gathered}
\forall i \sum_{l_{j k} \in\left\{x_{i}, \neg x_{i}\right\}} \boldsymbol{m}\left[l_{j k}\right] \geq 1 \\
\forall j, k, j^{\prime}, k^{\prime} l i t_{j k}=l i t_{j^{\prime} k^{\prime}} \Rightarrow \boldsymbol{m}\left[l_{j k}\right]=\boldsymbol{m}\left[l_{j^{\prime} k^{\prime}}\right]
\end{gathered}
$$

Now define $\nu$ by $\nu\left(x_{i}\right)=$ true if for some $l i t_{j k}=x_{i}, \boldsymbol{m}\left(l_{j k}\right)>0$. Due to the hypothesis, there is a clause $c_{j}$ such that $\nu\left(c_{j}\right)=$ false. Due to our choice of $\nu$ and the invariants, all inputs of $n c_{j}$ are marked. So firing $n c_{j}$ marks suc. 
We show that even the hypotheses that allow the lim-reachability to belong in $\mathrm{NC}$ do not reduce the complexity of other problems. The proof of this proposition is in the appendix.

Proposition 30 The (lim-)deadlock-freeness, (lim-)liveness and reversibility problems in consistent CPN systems with no initially empty siphons are coNP-hard.

\section{Conclusions}

In this work we have analysed the complexity of the most standard problems for continuous Petri nets. For almost all these problems, we have characterised their complexity class by designing new decision procedures and/or providing reductions to complete problems. We have also shown that the reachability set inclusion, undecidable for Petri nets, becomes decidable in the continuous framework. These results are summarised in Table 2 .

There are three fruitful possible extensions of this work. Other properties like coverability could be studied. A temporal logic provides a specification language for expressing properties. In Petri nets, the model checking problem lies on the boundary of decidability depending on the type of logics (branching versus linear, propositional versus evenemential). We want to investigate this problem for continuous Petri nets. Hybrid Petri nets encompass both discrete and continuous Petri nets. So it would be interesting to examine the complexity and decidability of standard problems for the whole class or some appropriate subclasses of this formalism.

Table 2. Complexity bounds

\begin{tabular}{|l|l|}
\hline \multicolumn{1}{|c|}{ Problems } & \multicolumn{1}{c|}{ Upper and lower bounds } \\
\hline (lim-)reachability & $\begin{array}{l}\text { PTIME-complete } \\
\text { in NC for lim-reachability (resp. reachability) } \\
\text { when all transitions are fireable at least once } \\
\text { (resp. and also in the reverse CPN) } \\
\text { and the net is consistent }\end{array}$ \\
\hline $\begin{array}{l}\text { (lim-)boundedness } \\
\text { PTIME-complete }\end{array}$ \\
\hline $\begin{array}{l}\text { (lim-)deadlock-freeness (lim-)liveness } \\
\text { set inclusion }\end{array}$ & $\begin{array}{l}\text { coNP-complete } \\
\text { coNP-hard even for free-choice CPNs } \\
\text { or for CPNs when all transitions are fireable at least once } \\
\text { and the net is consistent }\end{array}$ \\
\hline $\begin{array}{l}\text { in EXPTIME } \\
\text { coNP-hard even for reversibility in CPNs } \\
\text { when all transitions are fireable at least once } \\
\text { and the net is consistent }\end{array}$ \\
\hline
\end{tabular}

Acknowledgments. The authors would like to thank Jorge Júlvez and Manuel Silva for fruitful discussions on CPNs. 


\section{References}

1. D. Avis, K. Fukuda, and S. Picozzi. On canonical representations of convex polyhedra. In A. M. Cohen, X.-S. Gao, and N. Takayama, editors, Mathematical Software, Proceedings of the First International Congress of Mathematical Software, pages 350-360. World Scientific Publishing, 2002.

2. R. Bagnara, P. M. Hill, and E. Zaffanella. Not necessarily closed convex polyhedra and the double description method. Formal Aspects of Computing, 17(2):222-257, 2005.

3. M. P. Cabasino, C. Seatzu, C. Mahulea, and M. Silva. Fault diagnosis of manufacturing systems using continuous Petri nets. In Proceedings of the IEEE International Conference on Systems, Man and Cybernetics, Istanbul, Turkey, pages 534-539. IEEE, 2010.

4. B. Codenotti, M. Leoncini, and F. P. Preparata. The role of arithmetic in fast parallel matrix inversion. Algorithmica, 30(4):685-707, 2001.

5. R. David and H. Alla. Continuous Petri nets. In Proc. of the 8th European Workshop on Application and Theory of Petri Nets, pages 275-294, Zaragoza, Spain, 1987.

6. J. Desel and J. Esparza. Free Choice Petri Nets. Cambridge Tracts in Theoretical Computer Science 40, 1995.

7. J. Esparza and M. Nielsen. Decidability issues for Petri nets - a survey. Elektronische Informationsverarbeitung und Kybernetik, 30(3):143-160, 1994.

8. B. Gudiño-Mendoza, E. López-Mellado, and H. Alla. Modeling and simulation of water distribution systems using timed hybrid Petri nets. Simulation, 88(3):329 $347,2012$.

9. J. Júlvez, L. Recalde, and M. Silva. On reachability in autonomous continuous Petri net systems. In W. van der Aalst and E. Best, editors, $24^{\text {th }}$ Int. Conf. on Application and Theory of Petri Nets, volume 2679 of LNCS, pages 221-240, Eindhoven, The Netherlands, 2003. Springer.

10. C. H. Papadimitriou. Computational complexity. Addison-Wesley, Reading, Massachusetts, 1994

11. C. H. Papadimitriou and K. Steigliz. Combinatorial Optimization. Algorithms and Complexity. Dover publications, second edition, 1998.

12. L. Recalde, S. Haddad, and M. Silva. Continuous Petri nets: Expressive power and decidability issues. Int. Journal of Foundations of Computer Science, 21(2):235$256,2010$.

13. L. Recalde, E. Teruel, and M. Silva. Autonomous continuous $\mathrm{P} / \mathrm{T}$ systems. In J. Kleijn S. Donatelli, editor, Application and Theory of Petri Nets 1999, volume 1639 of $L N C S$, pages 107-126, 1999.

14. R. Ross-Leon, A. Ramirez-Trevino, J. A. Morales, and J. Ruiz-Leon. Control of metabolic systems modeled with timed continuous Petri nets. In ACSD/Petri Nets Workshops, volume 827 of CEUR Workshop Proceedings, pages 87-102, 2010.

15. C. Renato Vázquez, H. Y. Sutarto, R. K. Boel, and M. Silva. Hybrid Petri net model of a traffic intersection in an urban network. In Proceedings of the IEEE International Conference on Control Applications, CCA 2010, pages 658-664, Yokohama, Japan, 2010.

16. N. Zerhouni and H. Alla. Dynamic analysis of manufacturing systems using continuous Petri nets. In Proceedings of the IEEE International Conference on Robotics and Automation, Cincinnati, OH, USA; volume 2, pages 1070-1075, Los Alamitos, CA, USA, 1990. 


\section{A Appendix: additional proofs}

Proof. (that for all $\boldsymbol{m} \in \lim -\operatorname{RS}\left(\mathcal{N}, \boldsymbol{m}_{0}\right), \lim -\operatorname{RS}(\mathcal{N}, \boldsymbol{m}) \subseteq \lim -\operatorname{RS}\left(\mathcal{N}, \boldsymbol{m}_{0}\right)$ )

Let $\boldsymbol{m}^{\prime} \in \lim -\mathrm{RS}(\mathcal{N}, \boldsymbol{m})$. Due to theorem 20, there exists $\mathbf{v}, \mathbf{v}^{\prime} \in \mathbb{R}_{\geq 0}^{|T|}$ such that:

1. $\boldsymbol{m}=\boldsymbol{m}_{0}+\boldsymbol{C v}$ and $\boldsymbol{m}^{\prime}=\boldsymbol{m}+\boldsymbol{C \mathbf { v } ^ { \prime }}$

2. $\llbracket \mathbf{v} \rrbracket \in F S\left(\mathcal{N}, \boldsymbol{m}_{0}\right)$ and $\llbracket \mathbf{v}^{\prime} \rrbracket \in F S(\mathcal{N}, \boldsymbol{m})$

Thus $\boldsymbol{m}^{\prime}=\boldsymbol{m}_{0}+\boldsymbol{C}\left(\mathbf{v}+\mathbf{v}^{\prime}\right)$.

Due to proposition 17 , since $\llbracket \mathbf{v} \rrbracket \in F S\left(\mathcal{N}, \boldsymbol{m}_{0}\right)$ there exists a sequence $\sigma$ and a marking $\boldsymbol{m}^{*}$ such that $\boldsymbol{m}_{0} \stackrel{\sigma}{\longrightarrow} \boldsymbol{m}^{*}$ and $\llbracket \vec{\sigma} \rrbracket=\llbracket \mathbf{v} \rrbracket$ and $\llbracket \boldsymbol{m}^{*} \rrbracket=\llbracket \boldsymbol{m}_{0} \rrbracket \cup \bullet \llbracket \mathbf{v} \rrbracket$.

Since $\boldsymbol{m}=\boldsymbol{m}_{0}+\boldsymbol{C} \mathbf{v}, \llbracket \boldsymbol{m} \rrbracket \subseteq \llbracket \boldsymbol{m}^{*} \rrbracket$ and so $\llbracket \mathbf{v}^{\prime} \rrbracket \in F S\left(\mathcal{N}, \boldsymbol{m}^{*}\right)$. So $\llbracket \mathbf{v}^{\prime} \rrbracket \cup \llbracket \mathbf{v}^{\prime} \rrbracket \in$ $F S\left(\mathcal{N}, \boldsymbol{m}_{0}\right)$. Using in the other direction the characterization of theorem 20 with $\mathbf{v}+\mathbf{v}^{\prime}$, one gets $\boldsymbol{m}^{\prime} \in \lim -\operatorname{RS}\left(\mathcal{N}, \boldsymbol{m}_{0}\right)$.

Proof.(proposition 27) Due to propositions 23 and 25, we only have to prove that these problems are PTIME-hard. So we design a LOGSPACE reduction from the circuit value problem (a PTIME-complete problem [10]) to these problems.

A circuit $\mathcal{C}$ is composed of four kinds of gates: False, True, AND, OR. Each gate has an output. There is a single False gate and a single True gate and they have no inputs. Gates whose type is AND or OR have two inputs. Any input of a gate is connected to an output of another gate. Let the binary relation $\prec$ between the gates be defined by: $a \prec b$ if the output of $a$ is connected to an input of $b$. Then one requires that the transitive closure of $\prec$ is irreflexive. One of the gates of the circuit, out, is distinguished and its output is not the input of any gate. The value of the inputs and outputs of a circuit is defined inductively according to the relation $\prec$. The output of gate False (resp. True) is false (resp. true). The input of a gate is equal to the value of the output to which it is connected. The output of a gate AND or OR is obtained by applying its truth table to its inputs. The circuit value problem consists in determining the value of the output of gate out.

The reduction is done as follows: The gate True is modelled by a place $p_{\text {True }}$ initially containing a token. This is the only place initially marked. The gate False is modelled by a place $p_{\text {False }}$. Any gate $c$ of kind AND yields a place $p_{c}$ and a transition $t_{c}$ whose inputs and outputs is represented in Fig. 5(a) and any gate $c$ of kind OR yields a place $p_{c}$ and two transitions $t_{c 1}$ and $t_{c 2}$ whose inputs and outputs are represented in Fig. 5(b). Finally one adds the subnet represented in Fig. 6 with one transition clean $_{p}$ per place $p$ different from $p_{\text {out }}$. This reduction can be performed in LOGSPACE.

We prove by induction on $\prec$ that a transition $t_{c}$ (resp. $t_{c 1}$ or $t_{c 2}$ ) is enabled iff the gate $c$ of kind AND (resp. OR) has value true.

Assume that gate $c$ of kind AND has value false. Then one of its input say $a$ has value false. If $a$ is the gate False then $p_{a}$ is initially unmarked and cannot be marked since it has no input. If $a$ is a gate of kind AND then by induction on 
$\prec, t_{a}$ is never enabled and so $p_{a}$ will always be empty. If $a$ is a gate of kind OR then by induction on $\prec, t_{a 1}$ and $t_{a 2}$ are never enabled and so $p_{a}$ will always be empty. Thus whatever the case $t_{c}$ can never be enabled. The case of a gate $c$ of kind $\mathrm{OR}$ is similar.

Assume that gate $c$ of kind AND has value true. Then both its inputs say $a$ and $b$ have value true. If $a$ (resp. $b$ ) is the gate True then $p_{a}$ (resp. $p_{b}$ ) is initially marked. If $a$ (resp. $b$ ) is a gate of kind AND then by induction on $\prec, t_{a}$ (resp. $t_{b}$ ) can be enabled. If $a$ is a gate of kind OR then by induction on $\prec$, some $t_{a i}$ (resp. $t_{b i}$ ) can be enabled. Now consider the sequence $\boldsymbol{m}_{0} \stackrel{\sigma}{\longrightarrow} \boldsymbol{m}$ of proposition 17 w.r.t. $\operatorname{maxFS}\left(\mathcal{N}, \boldsymbol{m}_{0}\right)$. In $\boldsymbol{m}$, every place initially marked or output of a transition that belongs to $\operatorname{maxFS}\left(\mathcal{N}, \boldsymbol{m}_{0}\right)$ is marked. So $t_{c}$ is enabled in $\boldsymbol{m}$. The case of a gate $c$ of kind OR is similar.

Now observe that the total amount of tokens in the net can only be increased by transition grow and in this case place $p_{\text {out }}$ is unbounded. Since $p_{\text {out }}$ can contain tokens iff the value of gate out is true, we have proved that the CPN system is unbounded iff the gate out is true.

Finally let $\boldsymbol{m}$ be defined by $\boldsymbol{m}\left(p_{\text {out }}\right)=1$ and $\boldsymbol{m}(p)=0$ for all $p \neq p_{\text {out }}$. If the value of gate out is false then $p_{\text {out }}$ will never be marked and so $\boldsymbol{m}$ is not reachable. If the value of gate out is true then transition $t_{\text {out }}$ can be fired by some small amount say $0<\varepsilon \leq 1$. Then all the other places can be unmarked by transitions clean $_{p}$ followed by a finite number of firings of grow in order to reach $\boldsymbol{m}$. So $\boldsymbol{m}$ is (lim-)reachable iff the value of gate out is true.

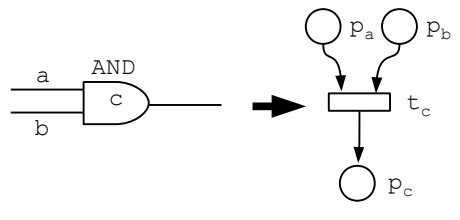

(a)

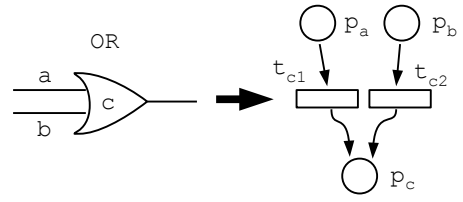

(b)

Fig. 5. Reductions of the gates (a) AND and (b) OR to CPN.

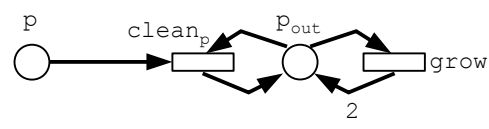

Fig. 6. An additional subnet.

Proof.(proposition 30) We use another reduction from the 3SAT problem already described in the proof of proposition 29 .

Every proposition $x_{i}$ yields a place $b_{i}$ initially marked with a token (all other places are unmarked) and input of two transitions: (1) $t_{i}$ with output place $p_{i}$ 
and, (2) $f_{i}$ with output place $n_{i}$ corresponding to the assignment associated with an interpretation. Every clause $c_{j}$ yields a transition $n c_{j}$. Transition $n c_{j}$ has three loop places corresponding to literals $l i t_{j k}$ : if $l i t_{j k}=x_{i}$ then the input is $n_{i}$, if $l i t_{j k}=\neg x_{i}$ then the input is $p_{i}$. An additional place $s u c$ is the output of transition $n c_{j}$. A transition $n d$ has $s u c$ for input place and no output place. Finally for every $x_{i}$, there are transitions $t b_{i}$ and $f b_{i}$ which are respectively reverse transitions of $t_{i}$ and $f_{i}$ with an additional loop over place suc. The reduction is illustrated in Fig. 7. The net is consistent with consistency vector: $\sum_{i}\left(t_{i}+t b_{i}+f_{i}+f b_{i}\right)+\sum_{j}\left(n c_{j}+n d\right)$. It does not contain an initially empty siphon since every siphon includes some place $b_{i}$. This proves that every transition can be fired at least once from $\boldsymbol{m}_{0}$.

Assume that there exists $\nu$ such that for all clause $c_{j}, \nu\left(c_{j}\right)=$ true. Then fire the following sequence $\sigma=1 t_{1}^{*} \ldots 1 t_{n}^{*}$ where $t_{i}^{*}=t_{i}$ when $\nu\left(x_{i}\right)=$ true and $t_{i}^{*}=f_{i}$ when $\nu\left(x_{i}\right)=$ false. Consider $\boldsymbol{m}$ the reached marking. Since $\nu\left(c_{j}\right)=$ true, at least one input place of $n c_{j}$ is empty in $\boldsymbol{m}$. Moreover $\boldsymbol{m}(s u c)=\boldsymbol{m}\left(b_{i}\right)=0$ for all $i$. So $\boldsymbol{m}$ is dead and the net is not reversible.

Assume that there does not exist $\nu$ such that for all clause $c_{j}, \nu\left(c_{j}\right)=$ true. Our goal is to prove that from any (lim-)reachable marking there is a sequence that comes back to $\boldsymbol{m}_{0}$. Since from $\boldsymbol{m}_{0}$ all transitions are fireable at least once this proves that the net is (lim-)live and (lim-)deadlock free.

For all atomic proposition $x_{i}$, and reachable marking $\boldsymbol{m}$, one has

$$
\forall i \boldsymbol{m}\left[b_{i}\right]+\boldsymbol{m}\left[p_{i}\right]+\boldsymbol{m}\left[n_{i}\right]=1
$$

Since a lim-reachable marking is a limit of reachable markings, this invariant also holds for lim-reachable markings.

If for some $i, \boldsymbol{m}\left[b_{i}\right]>0$, we fire $t_{i}$ in order to empty $b_{i}$. Thus the invariant becomes:

$$
\forall i \boldsymbol{m}\left[p_{i}\right]+\boldsymbol{m}\left[n_{i}\right]=1
$$

Now define $\nu$ by $\nu\left(x_{i}\right)=$ true if $\boldsymbol{m}\left(p_{i}\right)>0$. Due to the hypothesis, there is a clause $c_{j}$ such that $\nu\left(c_{j}\right)=$ false. Due to our choice of $\nu$ and the invariant, all inputs of $n c_{j}$ are marked. So firing $n c_{j}$ marks suc. Now fire transitions $t b_{i}$ and $f b_{i}$ in order to empty places $p_{i}$ and $n_{i}$. So $\boldsymbol{m}\left(b_{i}\right)=1$. Finally one fires $n d$ in order to empty place suc and we are done. 


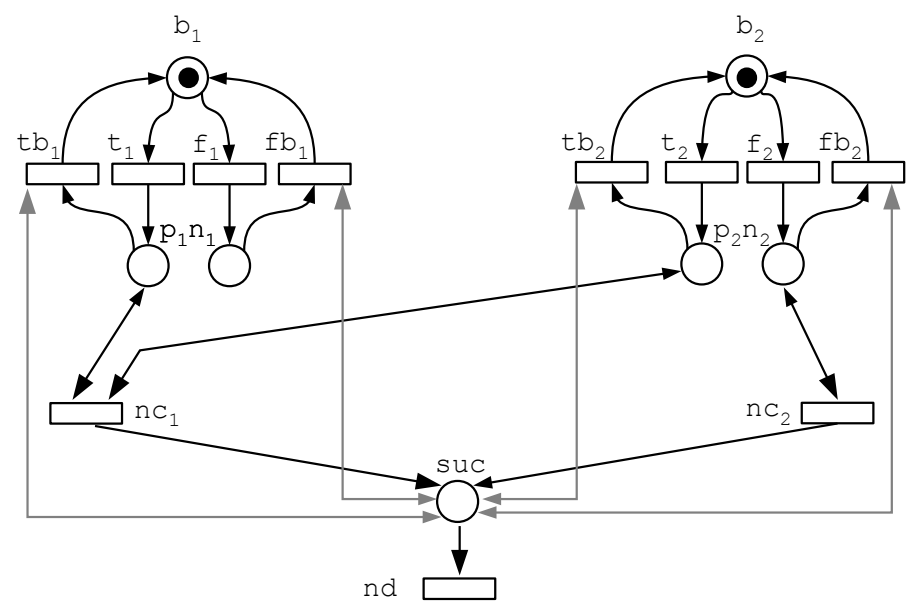

Fig. 7. The CPN corresponding to formula $\left(\neg x_{1} \vee \neg x_{2}\right) \wedge x_{2}$. 